\title{
Unified Importance Sampling Schemes for Efficient Simulation of Outage Capacity over Generalized Fading Channels
}

\author{
Nadhir Ben Rached, Abla Kammoun, Mohamed-Slim Alouini, and Raul Tempone
}

\begin{abstract}
The outage capacity (OC) is among the most important performance metrics of communication systems operating over fading channels. Of interest in the present paper is the evaluation of the $\mathrm{OC}$ at the output of the Equal Gain Combining (EGC) and the Maximum Ratio Combining (MRC) receivers. In this case, it can be seen that this problem turns out to be that of computing the Cumulative Distribution Function (CDF) for the sum of independent random variables. Since finding a closedform expression for the CDF of the sum distribution is out of reach for a wide class of commonly used distributions, methods based on Monte Carlo (MC) simulations take pride of price. In order to allow for the estimation of the operating range of small outage probabilities, it is of paramount importance to develop fast and efficient estimation methods as naive Monte Carlo (MC) simulations would require high computational complexity. In this line, we propose in this work two unified, yet efficient, hazard rate twisting Importance Sampling (IS) based approaches that efficiently estimate the OC of MRC or EGC diversity techniques over generalized independent fading channels. The first estimator is shown to possess the asymptotic optimality criterion and applies for arbitrary fading models, whereas the second one achieves the well-desired bounded relative error property for the majority of the well-known fading variates. Moreover, the second estimator is shown to achieve the asymptotic optimality property under the particular Log-normal environment. Some selected simulation results are finally provided in order to illustrate the substantial computational gain achieved by the proposed IS schemes over naive MC simulations.
\end{abstract}

Index Terms-Outage capacity, naive Monte Carlo, hazard rate twisting, Importance Sampling, asymptotic optimality, bounded relative error.

\section{INTRODUCTION}

Signal processing have for a long time had an important role to play in wireless communication systems. The nature of the wireless fading on the one hand and the ever-growing demands for higher data rates on the other have pushed together for the development of sophisticated signal processing algorithms. In order to mitigate the impact of severe fading, several algorithms, known as diversity techniques, have found the way into wireless communication systems. Among them, we distinguish the Maximum Ratio Combining (MRC) and the Equal Gain Combining (EGC), which are well-recognized for their ability

The authors are in Computer, Electrical and Mathematical Science and Engineering (CEMSE) Division, King Abdullah University of Science and Technology (KAUST), Thuwal, Makkah Province, Saudi Arabia , and also members of the KAUST Strategic Research Initiative on Uncertainty Quantification in Science and Engineering (SRI-UQ) (e-mail: nadhir.benrached, abla.kammoun, slim.alouini, raul.tempone@kaust.edu.sa).

Part of this work has been accepted at the IEEE International Symposium on Information Theory (ISIT'2015), Hong Kong, Jun. 2015 [1]. to combat fading and hence improve system performances [2]. A question of major practical interest that has been extensively studied over the last decades concerns the assessment of these techniques' performances when operating over realistic fading channels. Amongst the various performance metrics that can be used for that purpose, an important practical one is the Outage Capacity (OC), which measures the probability that the capacity falls below a certain threshold.

Interestingly, when MRC or EGC techniques are employed, the computation of the $\mathrm{OC}$ is equivalent to the evaluation of the Cumulative Distribution Function (CDF) of a sum of positive Random Variables (RVs) corresponding to either the sum of the fading envelopes (EGC) or the channel gains (MRC). For instance, under the Nakagami fading model, the OC is given by the CDF of the sum of Nakagami variates for the EGC technique and that of the sum of Gamma variates for the MRC technique.

In general, for the wide majority of the commonly used distributions, the CDF of the sum is unknown in closed-form. This is for instance the case of Log-normal distributions, which are widely used to model the shadowing effect in cellular communication systems [3] and the weak-to-moderate turbulence channel in free space optical communication systems [4]. This is also the case of Gamma-Gamma distributions, considered as the dominant fading model for free space optical links [5], and that of Weibull distributions known for their good fits to experimental fading channel measurements for both indoor and outdoor environment [6], [7]. All these practical contexts have stirred a great deal of interest in the development of approximation methods of the CDF of the sum distribution. Given the large amount of works in this context, it is difficult to provide an exhaustive survey of the existing techniques. However, we can mainly classify these approaches into two categories. The first category includes approaches where closed-form expressions are provided to approximate the CDF of the sum [8]-[14]. For instance, we can cite the works in [8] and [9] that approximate the sum of Log-normal RVs by a Log-normal and a Pearson type IV variates, respectively. Along the same line, the $\alpha-\mu$ PDF envelope was used to approximate the sum of Weibull [10], Nakagami [11], and Gamma-Gamma [12] variates. Although the previously cited works [8]-[14] were shown to exhibit a good level of accuracy, there is no guarantee that they maintain the same level of accuracy, whatever are the range of probabilities, the set of parameters distribution, and the number of summands [8]. A second class of techniques have 
been then proposed that are rather based on efficient numerical integration methods [15], [16]. In the same framework, a general numerical approach has been developed in [17] given the knowledge of the Moment Generating Function (MGF). It is worth mentioning that such a requirement is not ensured for many well-challenging distributions such as the Log-normal, the Nakagami, and the Weibull ones. Despite their popularity, the previously cited approximation techniques share the common drawback of being non generic, that is, specific to a given distribution (or a narrow class of distributions). This principally constitutes the major motivation behind the present work. In particular, in a completely different approach, we build upon the framework of rare event simulation algorithms in order to estimate the CDF of sums of arbitrary RVs (equivalently the OC of MRC/EGC diversity techniques operating over generalized fading channels). Despite the continuous advances in the development of these algorithms, their popularity among researchers in wireless communication systems has thus far remained limited. To the best of our knowledge, our work is the first one to bridge the gap between the framework of rare event algorithms and the OC evaluation of EGC/MRC diversity techniques.

As the quantity of interest corresponds to operating outage probabilities, the focus will be put on the left-tail of the sum distribution, i.e. the probability that the sum is below a given small threshold. It is worth mentioning that in the context of rare event algorithms, most of the existing approaches have thus far been focused on the right tail of the sum distribution (probability of the sum greater than a sufficiently large threshold) instead of the left-tail region (which is the region of interest of the present work). It is only recently that the authors in [18] and [19] have considered the left tail but their works concerned the specific setting of the sum of Log-normal RVs. More precisely, the approach in [18] was based on the well-known exponential twisting technique [20], [21] and was shown to achieve the asymptotic optimality property under the i.i.d assumption. However, as a derivative of the exponential twisting technique, the proposed approach presents the drawback of requiring the existence of a closedform expression for the MGF. Such a requirement cannot be met in the case of a Log-normal RV, and hence an estimator for the MGF was used instead [22].

An alternative approach that avoids the need for the MGF is represented by the hazard rate twisting technique [23][26]. This technique cannot be directly applied to our setting, since it was originally developed to deal with the righttail of the sum distribution. Based on an adjustment of this technique, we propose in this paper two unified Importance Sampling (IS) based approaches [27] that efficiently estimate the CDF of a sum of independent RVs (the left-tail of a sum distribution) with arbitrary distributions. The connection of this problem with that of the evaluation of the $\mathrm{OC}$ at the output of MRC/EGC techniques make the results of our work interesting to both fields of wireless communication and rare event simulation. While outperforming naive Monte Carlo (MC) simulations, the two proposed schemes exhibit different properties. As a matter of fact, the first proposed IS scheme applies to arbitrary independent positive distributions and was shown to enjoy the asymptotic optimality criterion. On the other hand, the second one satisfies the asymptotic optimality for the Log-normal setting. It also holds the sought-after stronger bounded relative error criterion for the majority of the well-known distributions but is only applicable to the case of i.i.d positive RVs. These two properties represent a relevant novelty in the context of rare event algorithms since, to the best of the authors knowledge, a unified IS scheme with the asymptotic optimality property for arbitrary sum distribution has not been proposed before when the left-tail of the sum distribution is considered. Moreover, and to the best of the authors knowledge, the second proposed IS approach is the first estimator to achieve the bounded relative error criterion when estimating the left tail region of the sum distribution, a property that represents one of the most valuable sought-after requirements of rare event simulation algorithms.

The rest of the paper is organized as follows. In Section II, we describe the problem setting and introduce the concept of IS. The methodologies adopted in our work are developed in Section III where two efficient IS schemes are proposed. In the same section, theorems showing their efficiency are also stated and proved. Some selected simulation results are provided in Section IV in order to illustrate the substantial computational gain achieved by the proposed IS approaches over naive MC simulations.

\section{Problem Setting}

\section{A. Motivation}

The instantaneous Signal-to-Noise Ratio (SNR) at the diversity receiver can be written as [28]

$$
\gamma_{e n d}=\frac{E_{s}}{N_{0} \sqrt{N^{1-p+q}}}\left(\sum_{i=1}^{N} R_{i}^{p}\right)^{q}
$$

where $N$ is the number of diversity branches, $E_{s} / N_{0}$ is the SNR per symbol at the transmitter, and $R_{i}, i=1,2, \ldots, N$, is the fading envelope, i.e. the fading channel amplitude or the modulus of the fading channel. The parameter $p$ and $q$ are in $\{1,2\}$ as follows

$$
(p, q)= \begin{cases}(1,2), & \mathrm{EGC} \\ (2,1), & \mathrm{MRC}\end{cases}
$$

The instantaneous capacity with MRC or EGC diversity techniques over fading channels has the following expression

$$
C_{\gamma_{e n d}}=W \log _{2}\left(1+\gamma_{e n d}\right),
$$

where $W$ is the bandwidth. Among the most important performance metrics of communication systems operating over fading channels is the OC. This metric is defined as the probability that the instantaneous capacity $C_{\gamma_{e n d}}$ falls below a certain threshold $C_{t h}$, that is

$$
C_{\text {out }}=P\left(0 \leq C_{\gamma_{\text {end }}}<C_{t h}\right) .
$$

This metric measures the probability that the communication system undergoes an outage and thus fails to operate. From 
(1) and (3), the OC can be rewritten as follows

$$
C_{\text {out }}=P\left(\sum_{i=1}^{N} R_{i}^{p}<\gamma_{t h}=\left(\frac{N_{0} \sqrt{N^{1-p+q}}}{E_{s}}\left(2^{\frac{C_{t h}}{W}}-1\right)\right)^{\frac{1}{q}}\right) .
$$

Hence, the computation of the OC with MRC or EGC diversity techniques boils down to the problem of determining the CDF of the sum of the fading envelopes (EGC), i.e. the CDF of $\sum_{i=1}^{N} R_{i}$, or the sum of the channel gains (MRC), i.e. the $\mathrm{CDF}$ of $\sum_{i=1}^{N} R_{i}^{2}$.

Many fading models are used in practice. A non exhaustive list includes for instance the Rayleigh, the Nakagami, the Weibull, the Rice, and the Log-normal fading models. The readers are referred to [29] for a more comprehensive list of the well practical fading models.

At a higher level of abstraction, our goal is then to develop efficient algorithms to estimate the quantity

$$
\alpha=P\left(S_{N}=\sum_{i=1}^{N} X_{i} \leq \gamma_{t h}\right),
$$

for a given threshold $\gamma_{t h}$, where $X_{1}, X_{2}, \ldots, X_{N}$ is a sequence of independent and not necessarily identically distributed positive RVs with Probability Density Function (PDF) $f_{X_{i}}(\cdot)$, $i=1,2, \ldots, N$.

\section{B. Importance Sampling}

In typical wireless communication systems, the quantity $\alpha$, which measures the OC, is relatively small. Thus, the focus will be put on estimating the probability of the rare event $\left\{\sum_{i=1}^{N} X_{i} \leq \gamma_{t h}\right\}$ when $\gamma_{t h}$ is relatively small. The naive Monte Carlo (MC) estimator of $\alpha$ is given by:

$$
\hat{\alpha}_{M C}=\frac{1}{M} \sum_{i=1}^{M} \mathbf{1}_{\left(S_{N}\left(\omega_{i}\right) \leq \gamma_{t h}\right)},
$$

where $M$ is the number of simulation runs, $\mathbf{1}_{(\cdot)}$ defines the indicator function, and $\left\{S_{N}\left(\omega_{i}\right)\right\}_{i=1}^{M}$ represent independent and identically distributed (i.i.d) realizations of the RV $S_{N}=$ $\sum_{j=1}^{N} X_{j}$ where for each realization, $i=1,2, \ldots, M$, the sequence $X_{1}\left(\omega_{i}\right), X_{2}\left(\omega_{i}\right), \ldots, X_{N}\left(\omega_{i}\right)$ are sampled independently according to the PDF $f_{X_{i}}(\cdot), i=1,2, \ldots, N$. In the setting of rare events simulations, i.e. events with very small probabilities, naive MC simulations are known to be computationally expensive. In fact, for an accuracy requirement of $90 \%$, it can be shown from the Central Limit Theorem that we need more than $100 / \alpha$ samples to estimate the quantity of interest $\alpha$.

Importance Sampling (IS) is a variance reduction technique that can overcome the failure of naive $\mathrm{MC}$ simulations and considerably reduce the computational work [27]. The basic idea of IS techniques is to construct an unbiased estimator of $\alpha$ with much smaller variance than the variance given by the naive MC estimator. IS techniques are based on performing a change of the probability measure, that is sampling is performed according to a new distribution instead of the original one. In fact, the quantity $\alpha$ can be rewritten as follows:

$$
\begin{aligned}
\alpha & =\mathbb{E}_{p}\left[\mathbf{1}_{\left(S_{N} \leq \gamma_{t h}\right)}\right]=\int_{\mathbb{R}^{N}} \mathbf{1}_{\left(S_{N} \leq \gamma_{t h}\right)} \prod_{i=1}^{N} f_{X_{i}}\left(x_{i}\right) d x_{1} d x_{2} \ldots d x_{N} \\
& =\int_{\mathbb{R}^{N}} \mathbf{1}_{\left(S_{N} \leq \gamma_{t h}\right)} L\left(x_{1}, x_{2}, \ldots, x_{N}\right) \prod_{i=1}^{N} g_{X_{i}}\left(x_{i}\right) d x_{1} d x_{2} \ldots d x_{N} \\
& =\mathbb{E}_{p^{*}}\left[\mathbf{1}_{\left(S_{N} \leq \gamma_{t h}\right)} L\left(X_{1}, X_{2}, \ldots, X_{N}\right)\right]
\end{aligned}
$$

where $\mathbb{E}_{p}[\cdot]$ and $\mathbb{E}_{p^{*}}[\cdot]$ are the expectations with respect to the probability measures $p$ and $p^{*}$ under which the PDFs of $X_{i}$ are $f_{X_{i}}(\cdot)$ and $g_{X_{i}}(\cdot), i=1,2, \ldots, N$, respectively. The likelihood ratio $L$ is defined as

$$
L\left(X_{1}, X_{2}, \ldots, X_{N}\right)=\prod_{i=1}^{N} \frac{f_{X_{i}}\left(x_{i}\right)}{g_{X_{i}}\left(x_{i}\right)}
$$

The idea behind performing this change of the sampling distribution is to emphasize the sampling of important values, i.e. values which have more impact on the parameter of interest $\alpha$ than others. More precisely, the change of the sampling distribution is performed in a way to increase the frequency of realizations belonging to the rare event $\left\{S_{N} \leq \gamma_{t h}\right\}$. Thus, by encouraging these important realizations, it is possible to achieve an interesting amount of variance reduction by using the IS estimator defined as:

$$
\hat{\alpha}_{I S}=\frac{1}{M} \sum_{i=1}^{M} \mathbf{1}_{\left(S_{N}\left(\omega_{i}\right) \leq \gamma_{t h}\right)} L\left(X_{1}\left(\omega_{i}\right), X_{2}\left(\omega_{i}\right), \ldots, X_{N}\left(\omega_{i}\right)\right),
$$

where for each realization $i=1,2, \ldots, M$, the sequence $X_{1}\left(\omega_{i}\right), X_{2}\left(\omega_{i}\right), \ldots, X_{N}\left(\omega_{i}\right)$ is now sampled according to the new PDFs $g_{X_{i}}(\cdot), i=1,2, \ldots, N$. Many criteria have been used in the literature of rare event algorithms in order to measure their efficiencies [30]. We call an IS estimator to possess the bounded relative error criterion if

$$
\limsup _{\gamma_{t h} \rightarrow 0} \frac{\mathbb{E}_{p^{*}}\left[\mathbf{1}_{\left(S_{N} \leq \gamma_{t h}\right)} L^{2}\left(X_{1}, X_{2}, \ldots, X_{N}\right)\right]}{\alpha^{2}}<\infty .
$$

An interesting feature of this property is that the number of simulation runs to achieve a fixed accuracy requirement remains bounded independently of how small is the probability of interest $\alpha$. This is the reason why the criterion of bounded relative error is considered as one of the most sought-after requirements of variance reduction algorithms. Generally, estimators with bounded relative error are difficult to construct. This often leads researchers to settle for estimators that satisfy weaker properties such as the asymptotic optimality (or the logarithmic efficiency) criterion [25] [23]. We say that $\alpha$ is asymptotically optimally estimated if

$$
\lim _{\gamma_{t h} \rightarrow 0} \frac{\log \left(\mathbb{E}_{p^{*}}\left[\mathbf{1}_{\left(S_{N} \leq \gamma_{t h}\right)} L^{2}\left(X_{1}, X_{2}, \ldots, X_{N}\right)\right]\right)}{\log (\alpha)}=2 .
$$

Note that this limit cannot be made larger, since, from the non-negativity of the variance of the RV $\mathbf{1}_{\left(S_{N} \leq \gamma_{t h}\right)} L\left(X_{1}, X_{2}, \ldots, X_{N}\right)$, we have

$$
\begin{aligned}
& \mathbb{E}_{p^{*}}\left[\mathbf{1}_{\left(S_{N} \leq \gamma_{t h}\right)} L^{2}\left(X_{1}, X_{2}, \ldots, X_{N}\right)\right] \\
& \geq\left(\mathbb{E}_{p^{*}}\left[\mathbf{1}_{\left(S_{N} \leq \gamma_{t h}\right)} L\left(X_{1}, X_{2}, \ldots, X_{N}\right)\right]\right)^{2}=\alpha^{2},
\end{aligned}
$$


and thus, applying the logarithm function on both sides and dividing by $\log (\alpha)<0$, we obtain

$$
\frac{\log \left(\mathbb{E}_{p^{*}}\left[\mathbf{1}_{\left(S_{N} \leq \gamma_{t h}\right)} L^{2}\left(X_{1}, X_{2}, \ldots, X_{N}\right)\right]\right)}{\log (\alpha)} \leq 2 .
$$

Equation (12) reveals an interesting interplay between $\alpha$ and the second moment $\mathbb{E}_{p^{*}}\left[\mathbf{1}_{\left(S_{N} \leq \gamma_{t h)}\right)} L^{2}\left(X_{1}, X_{2}, \ldots, X_{N}\right)\right]$. Indeed, one can see that when $\alpha^{2} \rightarrow 0$ at an exponential rate, the second moment $\mathbb{E}_{p^{*}}\left[\mathbf{1}_{\left(S_{N} \leq \gamma_{t h}\right)} L^{2}\left(X_{1}, X_{2}, \ldots, X_{N}\right)\right]$ converges to zero at the same exponential rate. This is the best possible rate of convergence, i.e. the second moment cannot converge faster due to the non-negativity of the variance of $\mathbf{1}_{\left(S_{N} \leq \gamma_{t h}\right)} L\left(X_{1}, X_{2}, \ldots, X_{N}\right)$ in (14). Moreover, the asymptotic optimality property means that the number of samples to meet a fixed accuracy requirement satisfies $\log (M)=$ $o\left(\log \left(\alpha^{-1}\right)\right)$ (or equivalently $M=\mathcal{O}\left(\alpha^{-\epsilon}\right)$ for all $\epsilon>0$ ). This must be compared to the naive MC simulations which require a number of runs of order $\alpha^{-1}$ in order to achieve the same accuracy requirement.

It is worth noticing that the naive MC simulation fails to estimate $\alpha$ asymptotically optimally since the limit in (12) is equal to 1 .

\section{Methodology}

The methodology that will be adopted in the present work is based on the hazard rate twisting technique [23]. This approach was originally developed to deal with the right tail, that is, to estimate the probability of the rare event $\left\{S_{N}>\gamma_{t h}\right\}$, where $\gamma_{t h}$ is sufficiently large and the components of $S_{N}$ are independent and identically distributed (i.i.d) subexponential RVs (such as the Log-normal distribution and the Weibull distribution with shape parameter less than 1). It was then generalized in [25] to deal with the right tail of sums of independent but not necessarily identically arbitrary RVs prior to being further improved in [26]. Interestingly, the hazard rate twisting approach has never been applied to the left tail. This constitutes the major motivation behind our work. In particular, building upon the hazard rate twisting approach, we propose in this work two unified IS algorithms for the quick, yet accurate, estimation of the left-tail of a sum distribution. The first estimator applies to arbitrary independent and not identically distributed positive RVs and will be shown to achieve the asymptotic optimality criterion, whereas the second one is only valid for the case of i.i.d RVs but will be proven to satisfy the well-desired bounded relative error property under a mild assumption which is satisfied by most of the well-known fading variates. This makes it, to the best of our knowledge, the first estimator possessing this criterion when the left tail of the sum distribution is considered. Moreover, for the particular Log-normal fading model, the second estimator will be shown also to achieve the asymptotic optimality property under the i.i.d setting.

To facilitate the understanding of the proposed methods, we shall first recall the principle underlying the hazard rate twisting technique [25]. This is an important step that will help the reader understand how we adapt the technique of [25] to the considered problem. Let us define the hazard rate of $X_{i}, i=1,2, \ldots, N$, as

$$
\lambda_{X_{i}}(x)=\frac{f_{X_{i}}(x)}{1-F_{X_{i}}(x)}, x>0
$$

where $F_{X_{i}}(\cdot)$ is the CDF of $X_{i}, i=1,2, \ldots, N$. We define also the hazard function as

$$
\begin{aligned}
\Lambda_{X_{i}}(x) & =\int_{0}^{x} \lambda_{X_{i}}(t) d t \\
& =-\log \left(1-F_{X_{i}}(x)\right), x>0
\end{aligned}
$$

From (15) and (16), the PDF of the RV $X_{i}, i=1,2, \ldots, N$, is related to the hazard rate and the hazard function as follows:

$$
f_{X_{i}}(x)=\lambda_{X_{i}}(x) \exp \left(-\Lambda_{X_{i}}(x)\right), x>0
$$

The hazard rate twisting change of measure [25] consists in twisting the hazard rate of each component $X_{i}, i=1,2, \ldots, N$, by a quantity $0 \leq \theta<1$

$g_{X_{i}}(x) \triangleq f_{X_{i}, \theta}(x)=(1-\theta) \lambda_{X_{i}}(x) \exp \left(-(1-\theta) \Lambda_{X_{i}}(x)\right)$.

Prior to presenting our proposed approaches, we shall provide some insights on the role of the hazard rate twisting change of measure in efficiently dealing with the estimation of the right tail of the sum distribution, i.e, the probability of the event $\left\{S_{N} \geq \gamma_{t h}\right\}$ where $\gamma_{t h}$ is sufficiently large. Note that the change of measure (18) by an amount of $0 \leq \theta<1$ shifts the value of the hazard rate from $\lambda_{X_{i}}(x)$ to $(1-\theta) \lambda_{X_{i}}(x)$, $i=1,2, \ldots, N$. This is equivalent according to (16) to a change of the complementary CDF expression from $\exp \left(-\Lambda_{X_{i}}(x)\right)$ to $\exp \left(-(1-\theta) \lambda_{X_{i}}(x)\right), i=1,2, \ldots, N$. As a consequence, the tail of the resulting distribution becomes much heavier to the right than the original one. This constitutes the key feature that underlies the efficiency of the twisted distribution in generating more samples in the set $\left\{S_{N}>\gamma_{t h}\right\}$ for sufficiently large values of $\gamma_{t h}$. To illustrate this point, we consider the case in which a RV $X$ follows a Weibull distribution with shape and scale parameters denoted by $k$ and $\beta$. One can easily prove that the twisted PDF is again a Weibull distribution with the same shape parameter but with a higher scale parameter given by $\beta /(1-\theta)^{1 / k}$. It is thus more heavier to the right than the original distribution. However, the feature of having a more heavier tail to the right is not suitable for the present setting, wherein the focus is on the evaluation of the probability of the set $\left\{S_{N} \leq \gamma_{t h}\right\}$ where $\gamma_{t h}$ taking small values. In order to provide more insights on the failure of the technique in [25], we plot in Fig. 1.a the twisted and the untwisted distribution of $S_{N}$. From this figure, it is clearly obvious that the hazard rate twisting technique [25] accords much more emphasis to samples belonging to the rare set $\left\{S_{N}>\gamma_{t h}\right\}$, for a sufficiently large $\gamma_{t h}$, to the detriment of samples belonging to the rare set $\left\{S_{N} \leq \gamma_{t h}\right\}$ for small values of $\gamma_{t h}$. In consequence, a crude application of the approach of [25] will probably worsen the computational efficiency compared to the naive MC simulations.

Remark 1. In the rest of the paper, all expectations under the IS change of measure will be denote by $\mathbb{E}_{\theta}[\cdot]$ instead of $\mathbb{E}_{p^{*}}[\cdot]$. 
(a)

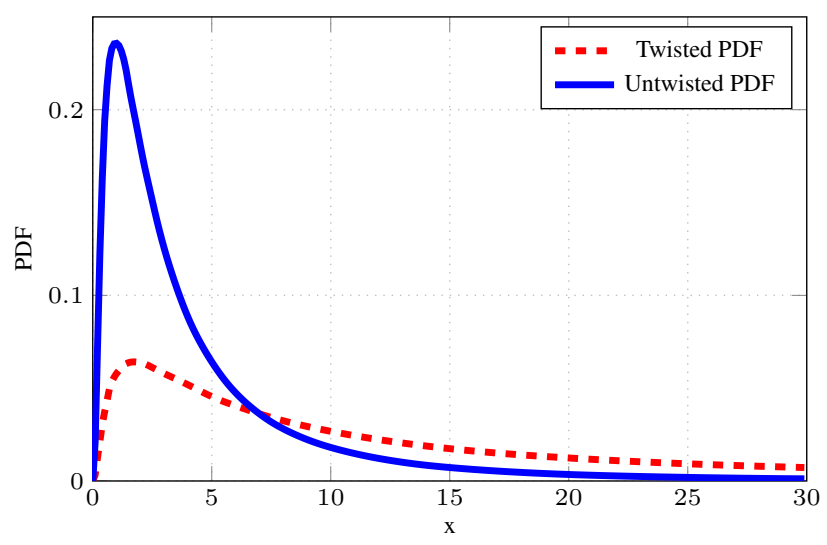

(b)

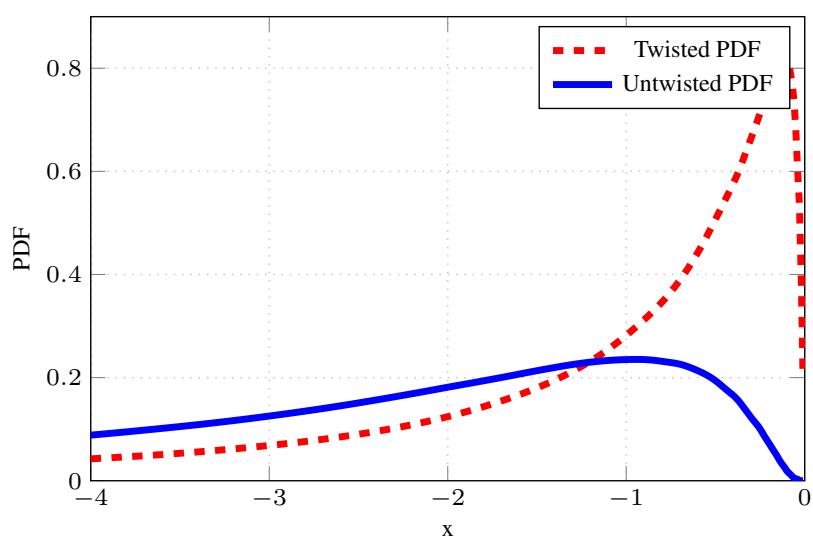

Fig. 1. Twisted and original PDFs: (a) PDF of $S_{2}$ with $X_{1}$ and $X_{2}$ are i.i.d Log-normal RVs with $\mu=0 \mathrm{~dB}, \sigma=6 \mathrm{~dB}$ and $\theta=0.6$, (b) PDF of $-S_{2}$ with $\theta=0.6$.

\section{A. First IS Approach}

For the hazard rate twisting technique to achieve a substantial computational gain, an adjustment is thus required. Our first IS estimator is based on the observation that the probability of interest corresponds in reality to the right tail of the distribution of $\sum_{i=1}^{N}-X_{i}$. As a matter of fact,

$$
\alpha=P\left(S_{N}=\sum_{i=1}^{N} X_{i} \leq \gamma_{t h}\right)=P\left(\sum_{i=1}^{N} Y_{i} \geq-\gamma_{t h}\right)
$$

where $Y_{i}=-X_{i}, i=1,2, \ldots, N$. It suffices thus to apply the hazard rate twisting technique to $Y_{i}$ instead of $X_{i}, i=1,2, \ldots, N$. The intuition behind this idea stems from the aforementioned feature of the hazard rate twisting technique of leading to a more heavier right-tail distribution than the underlying one. Thus, applying this technique to $Y_{i}, i=1,2, \ldots, N$ will certainly encourage the sampling of realizations in a neighborhood of 0 (since $Y_{i}, i=1,2, \ldots, N$, are non-negative), that is realizations belonging to the set $\left\{S_{N} \leq \gamma_{t h}\right\}$ for a sufficiently small $\gamma_{t h}$. This statement is clearly validated in Fig. 1.b showing the twisted and untwisted PDFs of $-S_{N}$. In this figure, we easily observe that the twisted PDF accords higher probability to samples belonging to the event of interest $\left\{\sum_{i=1}^{N} Y_{i} \geq-\gamma_{t h}\right\}$ (or equivalently
$\left\{S_{N}=\sum_{i=1}^{N} X_{i} \leq \gamma_{t h}\right\}$ ) for a sufficiently small $\gamma_{t h}$. Thus, we conclude that sampling with respect to the twisted PDF is more efficient than sampling with respect to the original one. Following the proposed adjustment, that is we apply the hazard rate twisting technique to the sequence $Y_{1}, Y_{2}, \ldots, Y_{N}$, the quantity of interest $\alpha$ is expressed following (8) as

$$
\begin{aligned}
\alpha & =\mathbb{E}_{p}\left[\mathbf{1}_{\left(S_{N} \leq \gamma_{t h}\right)}\right]=\int_{\mathbb{R}^{N}} \mathbf{1}_{\left(S_{N} \leq \gamma_{t h}\right)} \prod_{i=1}^{N} f_{Y_{i}}\left(y_{i}\right) d y_{1} d y_{2} \ldots d y_{N} \\
& =\mathbb{E}_{\theta}\left[\mathbf{1}_{\left(S_{N} \leq \gamma_{t h}\right)} L_{1}\left(Y_{1}, Y_{2}, \ldots, Y_{N}\right)\right]
\end{aligned}
$$

where $\mathbb{E}_{\theta}[\cdot]$ is now taken under the PDFs $g_{Y_{i}}(\cdot)$ obtained by twisting the hazard rate of the RV $Y_{i}, i=1,2, \ldots, N$, by $0 \leq \theta<1$. Similarly to (18), these twisted PDFs are defined as

$$
g_{Y_{i}}(y) \triangleq f_{Y_{i}, \theta}(y)=(1-\theta) \lambda_{Y_{i}}(y) \exp \left(-(1-\theta) \Lambda_{Y_{i}}(y)\right),
$$

where $\lambda_{Y_{i}}(y)=\frac{f_{X_{i}}(-y)}{F_{X_{i}}(-y)}$ and $\Lambda_{Y_{i}}(y)=-\log \left(F_{X_{i}}(-y)\right)$. The corresponding likelihood ratio is then expressed as:

$$
\begin{aligned}
L_{1}\left(Y_{1}, Y_{2}, \ldots, Y_{N}\right) & =\prod_{i=1}^{N} \frac{f_{Y_{i}}\left(Y_{i}\right)}{g_{Y_{i}}\left(Y_{i}\right)}=\prod_{i=1}^{N} \frac{f_{X_{i}}\left(-Y_{i}\right)}{g_{Y_{i}}\left(Y_{i}\right)} \\
& =\frac{1}{(1-\theta)^{N}} \exp \left(-\theta \sum_{i=1}^{N} \Lambda_{Y_{i}}\left(Y_{i}\right)\right)
\end{aligned}
$$

Finally, the IS estimator is then given by:

$$
\hat{\alpha}_{I S, 1}=\frac{1}{M} \sum_{i=1}^{M} T_{\gamma_{t h}, 1}\left(\omega_{i}\right)
$$

where $T_{\gamma_{t h}, 1}$ is defined as:

$$
T_{\gamma_{t h}, 1}=\mathbf{1}_{\left(S_{N} \leq \gamma_{t h}\right)} L_{1}\left(Y_{1}, Y_{2}, \ldots, Y_{N}\right)
$$

1) Optimal Minmax Twisting Parameter: The selection of $\theta$ follows the same steps of the minmax approach proposed in [25]. It requires first to construct an upper bound of the likelihood ratio and thus of the second moment of $T_{\gamma_{t h}, 1}$. The twisting parameter $\theta$ is then selected so that this upper bound is minimized. An upper bound of the likelihood ratio can be obtained by solving the following maximization problem $(P)$ :

$$
\begin{aligned}
& (P): \max _{Y_{1}, \ldots, Y_{N}} L_{1}\left(Y_{1}, Y_{2}, \ldots, Y_{N}\right) \\
& \quad \text { Subject to } \sum_{i=1}^{N} Y_{i} \geq-\gamma_{t h}, Y_{i}<0, \quad i=1, \ldots, N .
\end{aligned}
$$

From (22), it can be seen that the maximization problem $(P)$ is equivalent to the following minimization problem $\left(P^{\prime}\right)$

$$
\begin{aligned}
\left(P^{\prime}\right): \min _{Y_{1}, \ldots, Y_{N}} & \sum_{i=1}^{N} \Lambda_{Y_{i}}\left(Y_{i}\right) \\
\text { Subject to } & \sum_{i=1}^{N} Y_{i} \geq-\gamma_{t h}, Y_{i}<0, \quad i=1, \ldots, N .
\end{aligned}
$$


Let us denote by $Y_{1}^{*}, Y_{2}^{*}, \ldots, Y_{N}^{*}$ the solution of $\left(P^{\prime}\right)$. The second moment of $T_{\gamma_{t h}, 1}$ can be bounded by:

$$
\mathbb{E}_{\theta}\left[T_{\gamma_{t h}, 1}^{2}\right] \leq \frac{1}{(1-\theta)^{2 N}} \exp \left(-2 \theta \sum_{i=1}^{N} \Lambda_{Y_{i}}\left(Y_{i}^{*}\right)\right) .
$$

A natural selection of the twisting parameter $\theta$ is thus given by the one that minimizes the above upper bound. Through a simple computation, the optimal minmax twisting parameter is:

$$
\theta_{1}^{*}=1-\frac{N}{\sum_{i=1}^{N} \Lambda_{Y_{i}}\left(Y_{i}^{*}\right)} .
$$

Remark 2. Note that since $\Lambda_{Y_{i}}(\cdot)$ are increasing functions, $i=1,2, \ldots, N$, the inequality constraint in the minimization problem $\left(P^{\prime}\right)$ turns out to be satisfied with equality, that is:

$$
\sum_{i=1}^{N} Y_{i}^{*}=-\gamma_{t h}
$$

Remark 3. In general, solving the global solution of $\left(P^{\prime}\right)$ might require the need of complex optimization methods. However, for distributions with log-concave CDF, $\left(P^{\prime}\right)$ boils down to solving a convex problem and thus convex optimization algorithms can be employed. This property is actually satisfied for many challenging variates such as Log-normal, Weibull, and Gamma distributions [31].

2) Asymptotic Optimality: We prove in the next theorem that the IS estimator $T_{\gamma_{t h}, 1}$ satisfies the asymptotic optimality property:

Theorem 1. For any sum of independent positive RVs, the probability of interest $\alpha$ is asymptotically optimally estimated using the proposed hazard rate twisting IS approach with the twisting parameter $\theta_{1}^{*}$ given by (28). That is:

$$
\lim _{\gamma_{t h} \rightarrow 0} \frac{\mathbb{E}_{\theta_{1}^{*}}\left[T_{\gamma_{t h}, 1}^{2}\right]}{\log (\alpha)}=2 .
$$

Proof: By replacing the minmax optimal twisting parameter (28) into (27), it follows that

$$
\mathbb{E}_{\theta_{1}^{*}}\left[T_{\gamma_{t h}, 1}^{2}\right] \leq\left(\frac{A\left(\gamma_{t h}\right)}{N}\right)^{2 N} \exp \left(-2 A\left(\gamma_{t h}\right)+2 N\right),
$$

where $A\left(\gamma_{t h}\right)=\sum_{i=1}^{N} \Lambda_{Y_{i}}\left(Y_{i}^{*}\right)$. Upon applying the logarithm function on both sides, we get

$$
\log \left(\mathbb{E}_{\theta_{1}^{*}}\left[T_{\gamma_{t h}, 1}^{2}\right]\right) \leq 2 N\left(1+\log \left(\frac{A\left(\gamma_{t h}\right)}{N}\right)\right)-2 A\left(\gamma_{t h}\right) .
$$

On the other hand, using the inequality constraint of the minimization problem $\left(P^{\prime}\right)$, we obtain that

$$
\cap_{i=1}^{N}\left\{Y_{i} \geq Y_{i}^{*}\right\} \subset\left\{\sum_{i=1}^{N} Y_{i} \geq-\gamma_{t h}\right\} .
$$

Since $Y_{1}, \cdots, Y_{N}$ are independent, it follows that:

$$
\prod_{i=1}^{N} P\left(Y_{i}>Y_{i}^{*}\right) \leq \alpha,
$$

or equivalently,

$$
-A\left(\gamma_{t h}\right) \leq \log (\alpha)
$$

Since $\log (\alpha) \rightarrow-\infty$ as $\gamma_{t h} \rightarrow 0$, the quantity $A\left(\gamma_{t h}\right) \rightarrow+\infty$ as $\gamma_{t h} \rightarrow 0$. Now, we combine (32) and (35) to get, for a sufficiently small $\gamma_{t h}$, that

$$
\frac{\log \left(\mathbb{E}_{\theta_{1}^{*}}\left[T_{\gamma_{t h}, 1}^{2}\right]\right)}{\log (\alpha)} \geq \frac{2 N\left(1+\log \left(\frac{A\left(\gamma_{t h}\right)}{N}\right)\right)-2 A\left(\gamma_{t h}\right)}{-A\left(\gamma_{t h}\right)} .
$$

By taking the limit as $\gamma_{t h} \rightarrow 0$ in the previous inequality and using the fact that $A\left(\gamma_{t h}\right) \rightarrow+\infty$ as $\gamma_{t h} \rightarrow 0$, it follows that

$$
\lim _{\gamma_{t h} \rightarrow 0} \frac{\log \left(\mathbb{E}_{\theta_{1}^{*}}\left[T_{\gamma_{t h}, 1}^{2}\right]\right)}{\log (\alpha)} \geq 2 .
$$

Finally, by combining the previous inequality with (14), we deduce that the asymptotic optimality (12) is satisfied and hence the proof is concluded.

It is worth recalling that the left tail of the sum distribution has rarely been considered in the literature. The unique existing work of which we are aware deals with the specific setting of Log-normal sums [18]. To the best of our knowledge, the proposed estimator $T_{\gamma_{t h}, 1}$ is the first one that satisfies the asymptotic optimality criterion and, at the same time, applies to arbitrary positive distributions.

\section{B. Second IS approach}

In this section, we develop a second IS algorithm that, while being based again on the hazard rate twisting technique, satisfies the well-desired criterion of bounded relative error. Instead of twisting the RVs $Y_{1}, Y_{2}, \ldots, Y_{N}$ with a twisting parameter $\theta$ between 0 and 1, we propose to use a negative $\theta$ and apply the hazard rate twisting approach to the original variates $X_{1}, X_{2}, \ldots, X_{N}$. Following this methodology, the quantity $\alpha$ is rewritten as (8)

$$
\begin{aligned}
\alpha & =\mathbb{E}_{p}\left[\mathbf{1}_{\left(S_{N} \leq \gamma_{t h}\right)}\right]=\int_{\mathbb{R}^{N}} \mathbf{1}_{\left(S_{N} \leq \gamma_{t h}\right)} \prod_{i=1}^{N} f_{X_{i}}\left(x_{i}\right) d x_{1} d x_{2} \ldots d x_{N} \\
& =\mathbb{E}_{\theta}\left[\mathbf{1}_{\left(S_{N} \leq \gamma_{t h}\right)} L_{2}\left(X_{1}, X_{2}, \ldots, X_{N}\right)\right]
\end{aligned}
$$

where $\mathbb{E}_{\theta}[\cdot]$ denotes now the expectation taken under $g_{X_{i}}(\cdot)$, $i=1,2, \ldots, N$, in (18) with a negative twisting parameter, i.e. $\theta<0$. The corresponding likelihood ratio is

$$
\begin{aligned}
L_{2}\left(X_{1}, X_{2}, \ldots, X_{N}\right) & =\prod_{i=1}^{N} \frac{f_{X_{i}}\left(X_{i}\right)}{g_{X_{i}}\left(X_{i}\right)} \\
& =\frac{1}{(1-\theta)^{N}} \exp \left(-\theta \sum_{i=1}^{N} \Lambda_{X_{i}}\left(X_{i}\right)\right) .
\end{aligned}
$$

Finally, the IS estimator is defined as:

$$
\hat{\alpha}_{I S, 2}=\frac{1}{M} \sum_{i=1}^{M} T_{\gamma_{t h}, 2}\left(\omega_{i}\right),
$$

where

$$
T_{\gamma_{t h}, 2}=\mathbf{1}_{\left(S_{N} \leq \gamma_{t h}\right)} L_{2}\left(X_{1}, X_{2}, \ldots, X_{N}\right) .
$$


The intuition behind this methodology lies in that the use of a negative twisting parameter reverses the behavior of the hazard rate twisting method by making the distribution of the sum more concentrated on the left tail, thus generating more samples in the set $\left\{S_{N} \leq \gamma_{t h}\right\}$. To validate this intuition, we plot in Fig. 2 the twisted and the untwisted PDF of $S_{N}$ where the components are i.i.d Log-normal RVs. As expected, this figure shows that the twisted PDF accords higher probability to small values than the underlying PDF. Hence, compared to sampling according to the original PDF, it will increase the number of occurrences of samples belonging to the desired region, i.e. samples belonging to the set $\left\{S_{N} \leq \gamma_{t h}\right\}$ for a sufficiently small $\gamma_{t h}$.

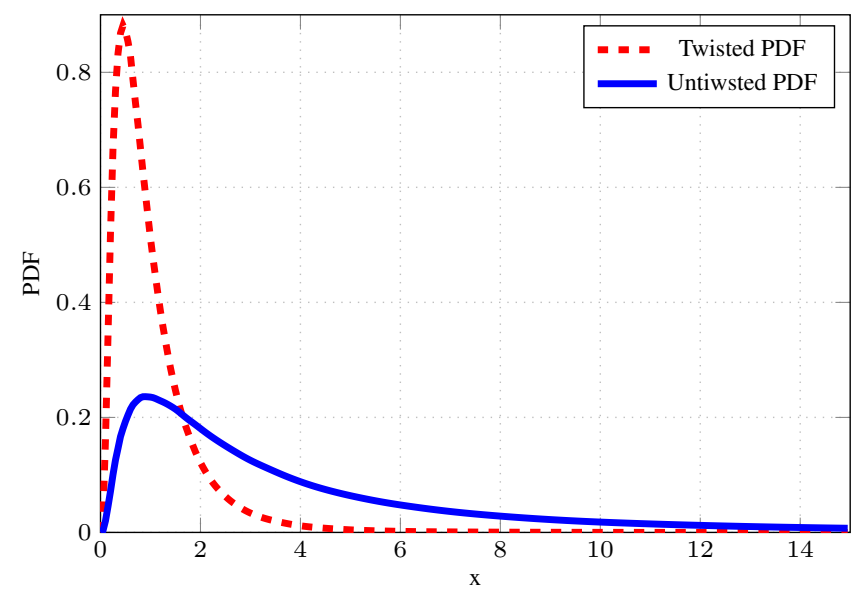

Fig. 2. Twisted and original PDFs of $S_{2}$ with $X_{1}$ and $X_{2}$ i.i.d Log-normal RVs with $\mu=0 \mathrm{~dB}, \sigma=6 \mathrm{~dB}$ and $\theta=-2$.

1) Minmax Optimal Parameter: The twisting parameter is determined through the minmax approach described in Section III-A. Since a negative $\theta$ is used, the minmax approach in the present setting involves the maximization of $\sum_{i=1}^{N} \Lambda_{X_{i}}\left(X_{i}\right)$ but over the different set $\left\{\sum_{i=1}^{N} X_{i} \leq \gamma_{t h}, X_{i}>0\right\}$ :

$$
\begin{aligned}
\left(P_{1}\right): \max _{X_{1}, \ldots, X_{N}} & \sum_{i=1}^{N} \Lambda_{X_{i}}\left(X_{i}\right) \\
& \text { Subject to } \sum_{i=1}^{N} X_{i} \leq \gamma_{t h}, X_{i}>0, \quad i=1, \ldots, N .
\end{aligned}
$$

This is obviously in contrast to the case of our first proposed IS method requiring to solve the minimization of $\sum_{i=1}^{N} \Lambda_{Y_{i}}\left(Y_{i}\right)$. Let $X_{1}^{*}, X_{2}^{*}, \ldots, X_{N}^{*}$ be the solution of $\left(P_{1}\right)$, hence we obtain:

$$
\mathbb{E}_{\theta}\left[T_{\gamma_{t h}, 2}^{2}\right] \leq \frac{1}{(1-\theta)^{2 N}} \exp \left(-2 \theta \sum_{i=1}^{N} \Lambda_{X_{i}}\left(X_{i}^{*}\right)\right) .
$$

Then, the value of $\theta$ is determined by minimizing the right hand side of the previous inequality which leads to

$$
\theta_{2}^{*}=1-\frac{N}{\sum_{i=1}^{N} \Lambda_{X_{i}}\left(X_{i}^{*}\right)} .
$$

2) Bounded Relative Error Property: We prove in the following theorem that the proposed IS scheme possesses the bounded relative error property. To the best of our knowledge, this is the first estimator of the left tail of the sum distribution (estimation of the probability of $\left\{S_{N} \leq \gamma_{t h}\right\}$ ) to achieve this criterion.

Theorem 2. Let $X_{1}, X_{2}, \ldots, X_{N}$ be a sequence of positive i.i.d RVs with a common hazard function $\Lambda(\cdot)$. Assume that $\Lambda\left(\gamma_{t h}\right)=\mathcal{O}\left(\Lambda\left(\gamma_{t h} / N\right)\right)$. Then, the bounded relative error property is achieved, that is,

$$
\limsup _{\gamma_{t h} \rightarrow 0} \frac{\mathbb{E}_{\theta_{2}^{*}}\left[T_{\gamma_{t h}, 2}^{2}\right]}{\alpha^{2}}<\infty .
$$

Proof: Plugging the minmax value of $\theta$ (44) into (43), we obtain:

$$
\left.\mathbb{E}_{\theta_{2}^{*}}\left[T_{\gamma_{t h}, 2}^{2}\right] \leq\left(\frac{1}{N} B\left(\gamma_{t h}\right)\right)\right)^{2 N} \exp \left(-2 B\left(\gamma_{t h}\right)+2 N\right),
$$

where $B\left(\gamma_{t h}\right)=\sum_{i=1}^{N} \Lambda\left(X_{i}^{*}\right)$. Given that $\Lambda(\cdot)$ is an increasing function and $X_{i}^{*} \leq \gamma_{t h}$ (this follows from the inequality constraint of the maximization problem $\left(P_{1}\right)$ ), we have that $B\left(\gamma_{t h}\right) \leq N \Lambda\left(\gamma_{t h}\right)$ and thus

$$
\mathbb{E}_{\theta_{2}^{*}}\left[T_{\gamma_{t h}, 2}^{2}\right] \leq\left(\Lambda\left(\gamma_{t h}\right)\right)^{2 N} \exp (2 N)
$$

On the other hand, we have

$$
\cap_{i=1}^{N}\left\{X_{i} \leq \gamma_{t h} / N\right\} \subset\left\{\sum_{i=1}^{N} X_{i} \leq \gamma_{t h}\right\},
$$

which leads, via the i.i.d assumption, to

$$
P\left(X_{1} \leq \gamma_{t h} / N\right)^{N}=\left(1-\exp \left(-\Lambda\left(\gamma_{t h} / N\right)\right)\right)^{N} \leq \alpha .
$$

Now, we combine the previous inequality with (47) to get

$$
\frac{\mathbb{E}_{\theta_{2}^{*}}\left[T_{\gamma_{t h}, 2}^{2}\right]}{\alpha^{2}} \leq \frac{\left(\Lambda\left(\gamma_{t h}\right)\right)^{2 N} \exp (2 N)}{\left(1-\exp \left(-\Lambda\left(\gamma_{t h} / N\right)\right)\right)^{2 N}}
$$

Using the fact that $\Lambda\left(\gamma_{t h}\right) \rightarrow 0$ as $\gamma_{t h} \rightarrow 0$, it follows that $1-\exp \left(-\Lambda\left(\gamma_{t h} / N\right)\right) \underset{\gamma_{t h} \rightarrow 0}{\sim} \Lambda\left(\gamma_{t h} / N\right)$, and thus we get

$$
\limsup _{\gamma_{t h} \rightarrow 0} \frac{\mathbb{E}_{\theta_{2}^{*}}\left[T_{\gamma_{t h}, 2}^{2}\right]}{\alpha^{2}} \leq \exp (2 N) \limsup _{\gamma_{t h} \rightarrow 0}\left(\frac{\Lambda\left(\gamma_{t h}\right)}{\Lambda\left(\gamma_{t h} / N\right)}\right)^{2 N}
$$

Finally, using the assumption $\Lambda\left(\gamma_{t h}\right)=\mathcal{O}\left(\Lambda\left(\gamma_{t h} / N\right)\right)$, we deduce that

$$
\limsup _{\gamma_{t h} \rightarrow 0} \frac{\mathbb{E}_{\theta_{2}^{*}}\left[T_{\gamma_{t h}, 2}^{2}\right]}{\alpha^{2}}<\infty,
$$

which concludes the proof.

It is worth mentioning that the assumption $\Lambda\left(\gamma_{t h}\right)=$ $\mathcal{O}\left(\Lambda\left(\gamma_{t h} / N\right)\right)$ does not cause a serious limitation, being satisfied by most of the well-known fading models. In practice, this assumption can be substituted by a more handy sufficient condition:

$$
\Lambda\left(\gamma_{t h}\right) \underset{\gamma_{t h} \rightarrow 0}{\sim} a \gamma_{t h}^{b}
$$


where $a$ is a constant and $b>0$. Moreover, since $\Lambda\left(\gamma_{t h}\right)=$ $-\log \left(1-F\left(\gamma_{t h}\right)\right) \underset{\gamma_{t h} \rightarrow 0}{\sim} F\left(\gamma_{t h}\right)$, the previous condition is equivalent to showing that:

$$
F\left(\gamma_{t h}\right) \underset{\gamma_{t h} \rightarrow 0}{\sim} a \gamma_{t h}^{b} .
$$

In Table I, we show that condition (54) holds for many wellknown challenging distributions. The results presented in the fourth column follows from the asymptotic behavior of the lower incomplete gamma function $\gamma(\cdot, \cdot)$ [32], the Marcum Q function $Q_{\xi}(\cdot, \cdot)$ [33], and the Meijer $\mathrm{G}$ function $G_{p, q}^{m, n}[\cdot]$ [34]. It is worth mentioning that these distributions correspond to the most used fading variates in practice, thereby showing the wide range of applicability of Theorem 2.

3) The Log-normal fading: Among the most commonly used distributions in the field of wireless communications, the Log-normal distribution is perhaps the sole one that we have found to not achieve the assumption of Theorem 2 . This does not imply however the unsuitability of our second IS to estimate the left tail of Log-normal sums. Indeed, our estimator, while we think it to not probably achieve the bounded relative error property, satisfies the asymptotic optimality criterion, as will be stated in the next theorem. Prior to stating the corresponding result, we shall recall that the Log-normal PDF is given by:

$$
f_{X_{i}}(x)=\frac{1}{\sqrt{2 \pi} x \sigma_{i}} \exp \left(-\frac{\left(\log (x)-\mu_{i}\right)^{2}}{2 \sigma_{i}^{2}}\right), x>0
$$

where $\mu_{i}$ and $\sigma_{i}, i=1,2, \ldots, N$, are respectively the mean and the standard deviation of the associated Gaussian RVs.

Theorem 3. The asymptotic optimality property is achieved when $X_{1}, X_{2}, \ldots, X_{N}$ is a sequence of i.i.d Log-normal RVs with a common hazard function $\Lambda(\cdot)$, that is we have

$$
\lim _{\gamma_{t h} \rightarrow 0} \frac{\log \left(\mathbb{E}_{\theta_{2}^{*}}\left[T_{\gamma_{t h}, 2}^{2}\right]\right)}{\log (\alpha)}=2 .
$$

Proof: In this proof, we will establish that our second IS holds the following equivalent characterization of the asymptotic optimality property [30]:

$$
\limsup _{\gamma_{t h} \rightarrow 0} \mathbb{E}_{\theta_{2}^{*}}\left[T_{\gamma_{t h}, 2}^{2}\right] / \alpha^{2-\epsilon}=0, \quad \forall \epsilon>0 .
$$

In order to prove (57), we note that using (47) and (49), we get for all $\epsilon>0$ :

$$
\begin{aligned}
\frac{\mathbb{E}_{\theta_{2}^{*}}\left[T_{\gamma_{t h}, 2}^{2}\right]}{\alpha^{2-\epsilon}} & =\mathcal{O}\left(\frac{\Lambda\left(\gamma_{t h}\right)^{2 N}}{\Lambda\left(\gamma_{t h} / N\right)^{N(2-\epsilon)}}\right) \\
& =\mathcal{O}\left(\frac{F\left(\gamma_{t h}\right)^{2 N}}{F\left(\gamma_{t h} / N\right)^{N(2-\epsilon)}}\right) .
\end{aligned}
$$

Now, we use an asymptotic equivalent for the CDF of the Log-normal distribution [35]

$$
\begin{aligned}
F\left(\gamma_{t h}\right) \underset{\gamma_{t h} \rightarrow 0}{\sim} \frac{\sigma}{\sqrt{2 \pi}\left(\log \left(1 / \gamma_{t h}\right)+\mu\right)} \\
\quad \times \exp \left(-\frac{\left(\log \left(1 / \gamma_{t h}\right)+\mu\right)^{2}}{2 \sigma^{2}}\right) .
\end{aligned}
$$

Using this result, we obtain:

$$
\begin{aligned}
& \frac{\mathbb{E}_{\theta_{2}^{*}}\left[T_{\gamma_{t h}, 2}^{2}\right.}{\alpha^{2-\epsilon}} \\
& =\mathcal{O}\left(\log \left(1 / \gamma_{t h}\right)^{-N \epsilon} \frac{\exp \left(-\frac{N\left(\log \left(1 / \gamma_{t h}\right)+\mu\right)^{2}}{\sigma^{2}}\right)}{\exp \left(-\frac{N(2-\epsilon)\left(\log \left(N / \gamma_{t h}\right)+\mu\right)^{2}}{2 \sigma^{2}}\right)}\right) \\
& \rightarrow 0 \text { as } \gamma_{t h} \rightarrow 0, \text { for all } \epsilon>0 .
\end{aligned}
$$

which ends up the proof.

Remark 4. We have shown that choosing the minmax parameter $\theta_{2}^{*}$ in (44) as the solution of the maximization problem $\left(P_{1}\right)$ guarantees the asymptotic properties proven in Theorem 2 and Theorem 3. However, a close look at the proof suggests another choice of the twisting parameter that not only avoids solving optimization problems but also keeps Theorem 2 and Theorem 3 satisfied. In fact, an upper bound of the second moment of $T_{\gamma_{t h}, 2}$, which is looser than the one obtained by solving the maximization problem $\left(P_{1}\right)$, is given by:

$$
\begin{aligned}
\mathbb{E}_{\theta}\left[T_{\gamma_{t h}, 2}^{2}\right] & \leq \mathbb{E}_{\theta}\left[\mathbf{1}_{\left(\cap_{i=1}^{N}\left\{X_{i} \leq \gamma_{t h}\right\}\right)} L\left(X_{1}, X_{2}, \ldots, X_{N}\right)\right] \\
& \leq \frac{1}{(1-\theta)^{2 N}} \exp \left(-2 \theta \sum_{i=1}^{N} \Lambda_{X_{i}}\left(\gamma_{t h}\right)\right)
\end{aligned}
$$

By setting the twisting parameter to be equal to:

$$
\theta_{2}^{*}=1-\frac{N}{\Lambda\left(\gamma_{t h}\right)},
$$

and by plugging this value into the (61), it follows, using the i.i.d assumption of Theorem 2 and 3, that

$$
\mathbb{E}_{\theta_{2}^{*}}\left[T_{\gamma_{t h}, 2}^{2}\right] \leq\left(\frac{\Lambda\left(\gamma_{t h}\right)}{N}\right)^{2 N} \exp \left(2 N^{2}\right)
$$

Hence, following the same steps as the original proofs of Theorem 2 and Theorem 3, we easily deduce that these two theorems holds with the choice of $\theta_{2}^{*}$ in (62). Based on extensive simulations, we have observed that the $\theta$ in (62) achieves approximately the same computational performances as those provided by the $\theta$ in (44), which makes it a good practical alternative that avoids the burden of optimization algorithms. The simulation results that will be presented in the next section correspond thus to the choice of $\theta$ in (62).

\section{Algorithm}

For the reader convenience, we provide hereafter a pseudo code describing the steps used by our first IS algorithm. 
Table I: Asymptotic Properties for Different Fading Channels ${ }^{a}$

\begin{tabular}{|l|c|c|c|}
\hline Fading Type & PDF & CDF & Asymptotic \\
\hline $\begin{array}{l}\text { Weibull } \\
k, \beta>0\end{array}$ & $\frac{k}{\beta}\left(\frac{x}{\beta}\right)^{k-1} \exp \left(-\left(\frac{x}{\beta}\right)^{k}\right)$ & $1-\exp \left(-\left(\frac{x}{\beta}\right)^{k}\right)$ & $\left(\frac{x}{\beta}\right)^{k}$ \\
\hline $\begin{array}{l}\text { Gamma, } k, \beta>0 \\
k, \beta>0\end{array}$ & $\frac{1}{\beta^{k} \Gamma(k)} x^{k-1} \exp \left(-\frac{x}{\beta}\right)$ & $\frac{1}{\Gamma(k)} \gamma\left(k, \frac{x}{\beta}\right)$ & $\frac{1}{\Gamma(k+1)}\left(\frac{x}{\beta}\right)^{k}$ \\
\hline $\begin{array}{l}\text { Nakagami, } m, \Omega>0 \\
m, \Omega>0\end{array}$ & $\frac{2 m^{m}}{\Gamma(m) \Omega^{m}} x^{2 m-1} \exp \left(-\frac{m}{\Omega} x^{2}\right)$ & $\frac{1}{\Gamma(m)} \gamma\left(m, \frac{m}{\Omega} x^{2}\right)$ & $\frac{1}{\Gamma(m+1)}\left(\frac{m}{\Omega} x^{2}\right)^{m}$ \\
\hline $\begin{array}{l}\text { Rice, } \sigma>0, \nu \geq 0 \\
\sigma>0, \nu \geq 0\end{array}$ & $\frac{x}{\sigma^{2}} \exp \left(-\frac{x^{2}+\nu^{2}}{2 \sigma^{2}}\right) I_{0}\left(\frac{x \nu}{\sigma^{2}}\right)$ & $1-Q_{1}\left(\frac{\nu}{\sigma}, \frac{x}{\sigma}\right)$ & $\frac{\exp \left(-\frac{\nu^{2}}{2 \sigma^{2}}\right)}{2 \sigma^{2}} x^{2}$ \\
\hline $\begin{array}{l}\text { Non-Centered Chi Squared } \\
k, \beta>0\end{array}$ & $\frac{\exp \left(-\frac{x+\beta}{2}\right)}{2}\left(\frac{x}{\beta}\right)^{\frac{k}{4}-\frac{1}{2}} I_{\frac{k}{2}-1}(\sqrt{\beta x})$ & $1-Q_{\frac{k}{2}}(\sqrt{\beta}, \sqrt{x})$ & $\frac{\exp \left(-\frac{\beta}{2}\right)}{\Gamma\left(\frac{k}{2}+1\right)}\left(\frac{x}{2}\right)^{\frac{k}{2}}$ \\
\hline $\begin{array}{l}\text { Gamma-Gamma } \\
m, k, \Omega>0\end{array}$ & $\frac{2(k m)^{\frac{k+m}{2}}}{\Gamma(k) \Gamma(m) \Omega}\left(\frac{x}{\Omega}\right)^{\frac{k+m}{2}-1} K_{k-m}\left(2 \sqrt{\frac{k m}{\Omega} x}\right)$ & $\frac{1}{\Gamma(m) \Gamma(k)} G_{1,3}^{2,1}\left[\left.\frac{k m}{\Omega} x\right|_{k, m, 0} ^{1}\right]$ & $\frac{\Gamma(m-k) \Gamma(k)}{\Gamma(1+k)} x^{k}$ \\
\hline
\end{tabular}

${ }^{a}$ Functions $\Gamma(\cdot), I_{\xi}(\cdot), K_{\xi}(\cdot)$ are respectively the Gamma function, the modified Bessel function of the first kind and order $\xi$, and the modified Bessel function of the second kind and order $\xi$ [32].

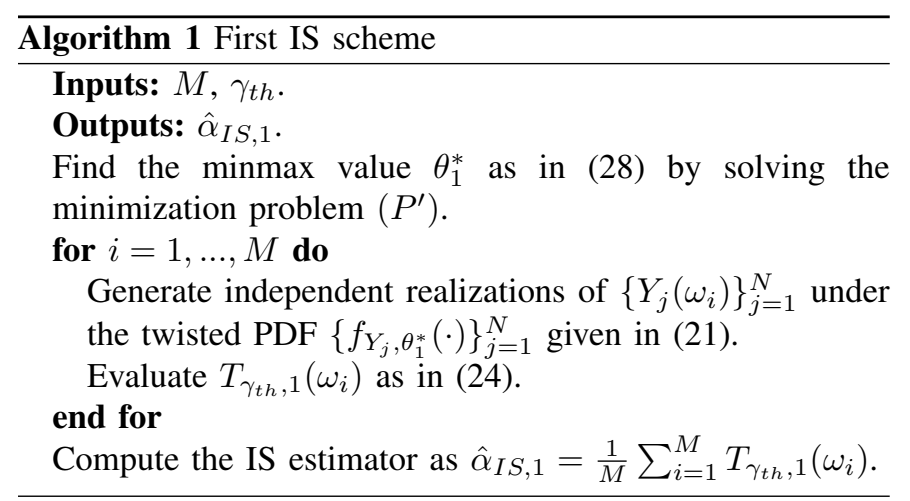

It is worth mentioning that these steps remain the same for the second IS scheme, the only differences being in the replacement of $\theta_{1}^{*}$ by $\theta_{2}^{*}$ given in $(62),\left\{f_{Y_{j}, \theta_{1}^{*}}(\cdot)\right\}_{j=1}^{N}$ by $\left\{f_{X_{j}, \theta_{2}^{*}}(\cdot)\right\}_{j=1}^{N}$ in (18), and $T_{\gamma_{t h}, 1}$ by $T_{\gamma_{t h}, 2}$ in (41).

Note that the implementation of Algorithm 1 requires generating new RVs according to the twisted PDFs $\left\{f_{Y_{i}, \theta}(\cdot)\right\}_{i=1}^{N}$. For that, several methods can be used, such as the inverse CDF method [36] which we elaborate on for the sake of completeness. Denoting the CDFs of $\left\{Y_{i}\right\}_{i=1}^{N}$ under the PDFs $\left\{f_{Y_{i}, \theta}(\cdot)\right\}_{i=1}^{N}$ by $\left\{F_{Y_{i}, \theta}(\cdot)\right\}_{i=1}^{N}$, the inverse CDF method is based on the observation that the $\operatorname{RV} F_{Y_{i}, \theta}^{-1}(U)$, where $U$ is uniformly distributed over $[0,1]$, has the same distribution as $Y_{i}$ under the twisted PDF $f_{Y_{i}, \theta}(\cdot), i=1,2, \ldots, N$. Using the expression of $f_{Y_{i}, \theta}(\cdot), i=1,2, \ldots, N$, given in (21) and after simple computations, we get a closed-form expression of the CDF inverse $F_{Y_{i}, \theta}^{-1}(\cdot), i=1,2, \ldots, N$, which is given by:

$$
F_{Y_{i}, \theta}^{-1}(u)=-F_{X_{i}}^{-1}\left((1-u)^{-\frac{1}{\theta-1}}\right) \text {. }
$$

In the second IS approach, where we need to sample from $\left\{f_{X_{i}, \theta_{2}^{*}}(\cdot)\right\}_{i=1}^{N}$ in (18), the CDF inverse is given as follows:

$$
F_{X_{i}, \theta}^{-1}(u)=F_{X_{i}}^{-1}\left(1-(1-u)^{-\frac{1}{\theta-1}}\right) .
$$

It is worth mentioning that the inverse CDF method is widely applicable to most of the well-known distributions, for which closed-form expressions of the CDF inverse exist. We can for instance cite the Log-normal, the Weibull, the Nakagami, and the Gamma distributions. Note also that, in case the inverse CDF is not easy to handle, one can use instead other sampling algorithms such as the acceptance-rejection technique and the Markov Chain Monte Carlo algorithm [37].

\section{Simulation Results}

In this section, we present the results of two simulation experiments illustrating the computational efficiency achieved by both of the proposed IS schemes. In the first experiment, we consider the evaluation of the $\mathrm{OC}$ at the output of the MRC receiver when operating over the Log-normal fading. The use of the Log-normal setting will allow us to compare with the simulation method in [18] which represents the sole simulation technique of which we are aware that deals with the left tail of the sum distribution. As a further application, we consider in the second experiment the evaluation of the $\mathrm{OC}$ at the output of the EGC receiver when operating over the Nakagami fading.

In both experiments, the gain in computational efficiency will be evaluated based on the amount of variance reduction over naive MC simulations that they achieve. For the consid- 
ered schemes, the amount of variance reduction writes as:

$$
k_{i}=\frac{\alpha(1-\alpha)}{\operatorname{var}_{\theta_{i}^{*}}\left[T_{\gamma_{t h}, i}\right]}, i=1,2 .
$$

The relevance of this metric lies in that it unveils the gain in simulations runs (over naive MC techniques) that can be achieved while maintaining a fixed accuracy requirement. More specifically, the naive $\mathrm{MC}$ simulations would require $k_{1} \times M$ (respectively $k_{2} \times M$ ) simulation runs to achieve the same accuracy as the first proposed IS technique (respectively the second IS technique ) when using $M$ simulation runs.

\section{A. Log-normal Fading with MRC Diversity Technique}

In this experiment, we evaluate the $\mathrm{OC}$ at the output of the MRC receiver when operating over the Log-normal fading. The PDF of the Log-normal envelope $R_{i}, i=1,2, \ldots, N$, is given in (55). It can be shown that the $\mathrm{OC}$ is given in this case by the CDF of the sum distribution of Log-normal RVs with parameters $2 \mu_{i}$ and $2 \sigma_{i}, i=1,2, \ldots, N$ where $\mu_{i}$ and $\sigma_{i}$ are the parameters corresponding to the distribution of the fading envelope $R_{i}$. The use of the log-normal setting allows us to compare our method with the IS estimator of [18], which we denote by $\hat{\alpha}_{I S, 3}=1 / M \sum_{i=1}^{M} T_{\gamma_{t h}, 3}\left(\omega_{i}\right)$ where $T_{\gamma_{t h}, 3}$ is given by [18, eq. 4.3]. This forces us to consider the i.i.d assumption, being required in [18]. Similarly to (66), we introduce for this approach the amount of variance reduction given by:

$$
k_{3}=\frac{\alpha(1-\alpha)}{\operatorname{var}_{\theta_{3}^{*}}\left[T_{\gamma_{t h}, 3}\right]},
$$

where $\theta_{3}^{*}$ is selected as in [18, eq. 2.5].

It is worth mentioning that the variances $T_{\gamma_{t h}, i}, i=1,2,3$ appearing in (66) and (67) are estimated using the empirical sample variances of $T_{\gamma_{t h}, i}, i=1,2,3$. Note that the use of the empirical sample variances makes sense for the proposed IS approaches, since we do have enough realizations in the set $\left(S_{N} \leq \gamma_{t h}\right)$ where $S_{N}$ represents the sum of the twisted RVs. The same calculation method cannot obviously be applied to estimate the variance introduced by the naive MC method, i.e. the variance of $\mathbf{1}_{\left(S_{N} \leq \gamma_{t h}\right)}$ under the underlying distribution. However, since it is in theory equal to $\alpha(1-\alpha)$, we proposed to approximate it by $\hat{\alpha}_{I S, i}\left(1-\hat{\alpha}_{I S, i}\right)$, where $\hat{\alpha}_{I S, i}, i=1,2,3$, are the IS estimators produced respectively by the two proposed IS schemes and the IS approach of [18].

It is also important to note that in this experiment, the twisting parameters $\theta_{1}^{*}$ in (28) and $\theta_{2}^{*}$ in (62) used by our first and second proposed IS schemes turn out to admit closedform expressions. As shown by (28), the value of $\theta_{1}^{*}$ requires solving the minimization problem $\left(P^{\prime}\right)$. Interestingly, using the log-concavity of the CDF of the Log-normal distribution [31] and under the i.i.d setting, a closed-form expression of the solution of $\left(P^{\prime}\right)$ can be easily obtained and corresponds to $Y_{i}^{*}=-\gamma_{t h} / N, i=1,2, \ldots, N$. Hence, a closed-form expression of $\theta_{1}^{*}$ is given by:

$$
\theta_{1}^{*}=1-\frac{1}{\Lambda_{Y_{1}}\left(-\gamma_{t h} / N\right)}
$$

(a)

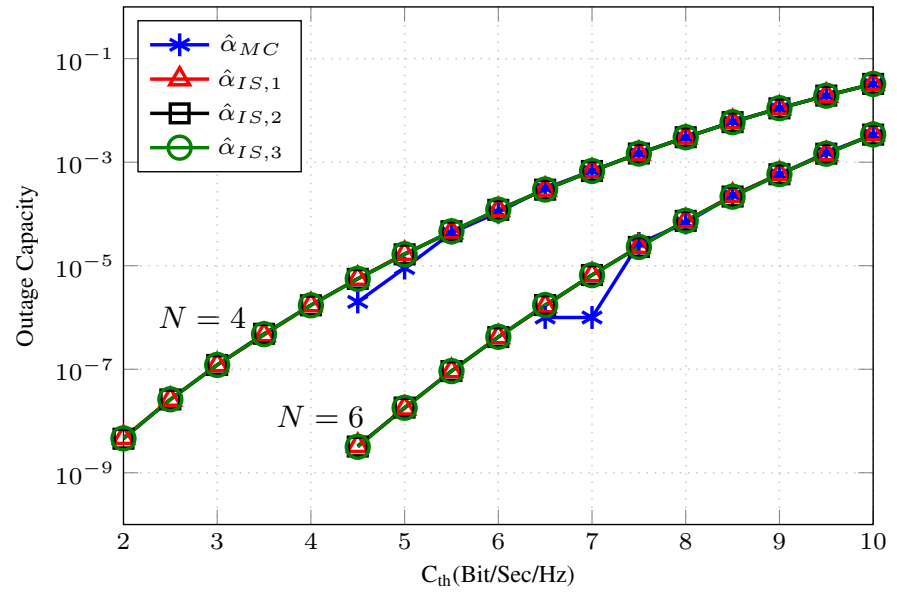

(b)

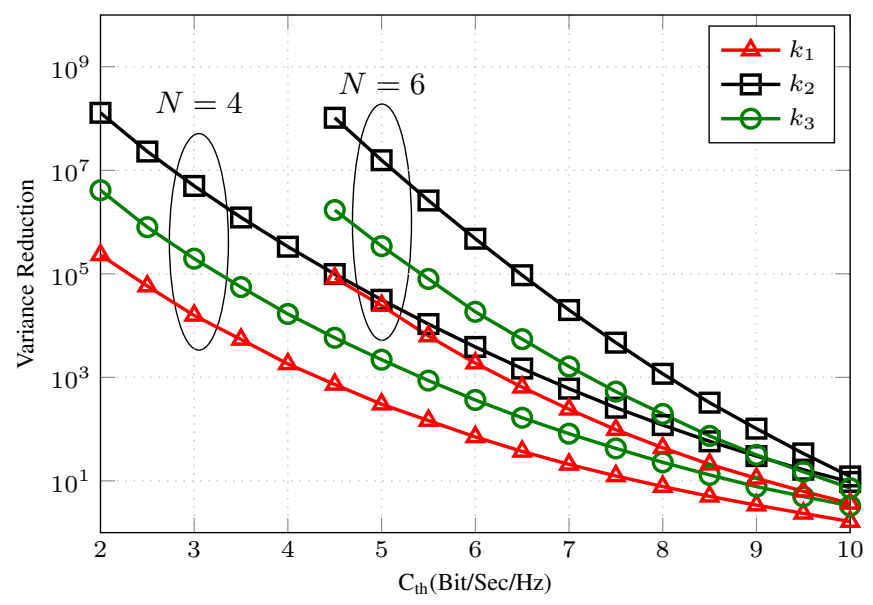

Fig. 3. (a) Outage capacity of $N$-branch MRC diversity receivers over i.i.d Log-normal fading variates with $E_{s} / N_{0}=10 \mathrm{~dB}, \mu=10 \mathrm{~dB}$, and $\sigma=6 \mathrm{~dB}$. (b) Efficiency $k_{i}, i=1,2,3$, with same parameters. Number of simulation runs: $M=10^{6}$.

where $\Lambda_{Y_{1}}(\cdot)$ is the hazard function of $Y_{1}, Y_{2}, \ldots, Y_{N}$ which is equal to

$$
\Lambda_{Y_{1}}(y)=-\log \left(\Phi\left(\frac{\log (-y)-2 \mu}{2 \sigma}\right)\right), \quad y<0,
$$

where $\Phi(\cdot)$ is the CDF of the standard normal distribution. For the second proposed IS scheme, the value of its twisting parameter $\theta_{2}^{*}$ is given in closed-form by (62). For the particular Log-normal fading environment with MRC diversity technique, it thus writes as:

$$
\theta_{2}^{*}=1+\frac{N}{\log \left(Q\left(\frac{\log \left(\gamma_{t h}\right)-2 \mu}{2 \sigma}\right)\right)},
$$

where $Q(\cdot)$ is the complementary $\mathrm{CDF}$ of the standard normal distribution.

In Fig. 3.a the outage capacity of $N$-branch MRC diversity receivers over independent Log-normal fading variates is estimated using the naive MC simulations, as well as the two proposed hazard rate twisting IS based approaches and the IS scheme of [18]. The number of simulation runs is $M=10^{6}$. From this figure, the failure of the naive MC 
simulation is clearly obvious. In fact, for each value of $N$, a clear oscillatory behavior is first observed for small values of the OC. Then, as we further decrease the threshold $C_{t h}$, it becomes unable to generate important samples, i.e. realizations that falls below the given threshold, and hence produces identically zero estimates. Such a failure can be avoided by using much more number of simulation runs. In sharp contrast to the behaviour of the naive MC estimator, both of the two proposed IS approach and the approach in [18] provide a smoother curve, thus revealing their higher accuracies while using the same number of samples.

We further proceed investigating the gain in terms of simulation runs that is achieved by both of the proposed IS methods and that of [18]. To this end, we plot in Fig. 3.b the amounts of variance reduction $k_{i}, i=1,2,3$ as a function of the capacity threshold $C_{t h}$. This figure clearly shows the high gain achieved by the three IS methods over naive MC simulations. This gain becomes all the more important as the threshold $C_{t h}$ decreases (small values of the OC). For instance, it can be seen from Fig. 3.b that for a fixed accuracy requirement, the naive $\mathrm{MC}$ simulations requires approximately $10^{8}$ times as many simulation runs as needed by the second IS approach to estimate the OC at the output of a 4 branch MRC receiver for $C_{t h}=2 \mathrm{Bit} / \mathrm{Sec} / \mathrm{Hz}$. Furthermore, Fig. 3.b shows that out of the three IS schemes and for the considered range of probabilities, it is the second IS scheme that exhibits the highest performance, requiring for $N=4$ and $C_{t h}=2$ $\mathrm{Bit} / \mathrm{Sec} / \mathrm{Hz}$, approximately $10^{3}$ and 10 times less simulation runs than our first proposed IS and the estimator in [18], respectively. Nevertheless, it is worth mentioning that the good performances of the second IS estimator and that of [18] must be balanced with their narrower scope of applicability. This is to be compared to the first IS estimator which applies to the general case of arbitrary independent and not necessarily identically distributed RVs.

In Fig. 4, we consider the evaluation of the $\mathrm{OC}$ at the output of $N=4$ branch MRC diversity receiver operating over independent Log-normal fading. Two scenarios are investigated that correspond to different choices of the values of the standard deviation $\sigma: \sigma=4 \mathrm{~dB}$ for the first scenario and $\sigma=5 \mathrm{~dB}$ for the second scenario. Fig. 4.a represents the OCs estimated for both scenarios by the naive MC simulations as well as the two proposed IS schemes and that of [18] when $M=10^{6}$. For both scenarios, we note that the three IS exhibit again a good level of accuracy, whereas the naive MC fails to be accurate using the same number of simulation runs. We further our analysis in Fig. 4.b by studying the computational gain that is achieved by the three IS approaches over MC simulations as a function of $C_{t h}$. This gain is expressed in terms of number of simulation runs and corresponds to the amount of variance reduction $k_{i}, i=1,2,3$. Fig. 4.b confirms the same conclusion as the previous experiment, in that for both choices of the standard deviation, all the considered IS schemes outperform naive MC simulations. The gain is all the more high as the threshold $C_{t h}$ take small values. For instance, for $\sigma=5 \mathrm{~dB}$ and $C_{t h}=3 \mathrm{Bit} / \mathrm{Sec} / \mathrm{Hz}$, the naive $\mathrm{MC}$ method requires above $10^{8}$ times more simulation runs than those used by our second IS technique in order to ensure the same level of accuracy. Moreover, the same figure confirms that our second IS approach achieves again better performances than the first proposed IS scheme and the one of [18]. We also note that the gain of the second IS scheme is less important for $\sigma=4 \mathrm{~dB}$ than that of $\sigma=6 \mathrm{~dB}$ shown in Fig. 3.b. We conclude thus that the second IS scheme is likely to achieve higher gains as $\sigma$ increases.

(a)

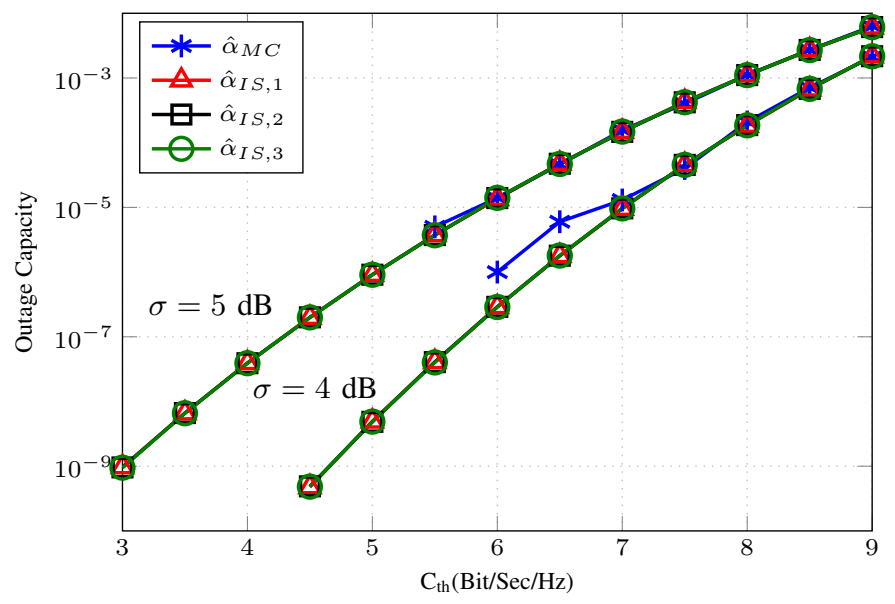

(b)

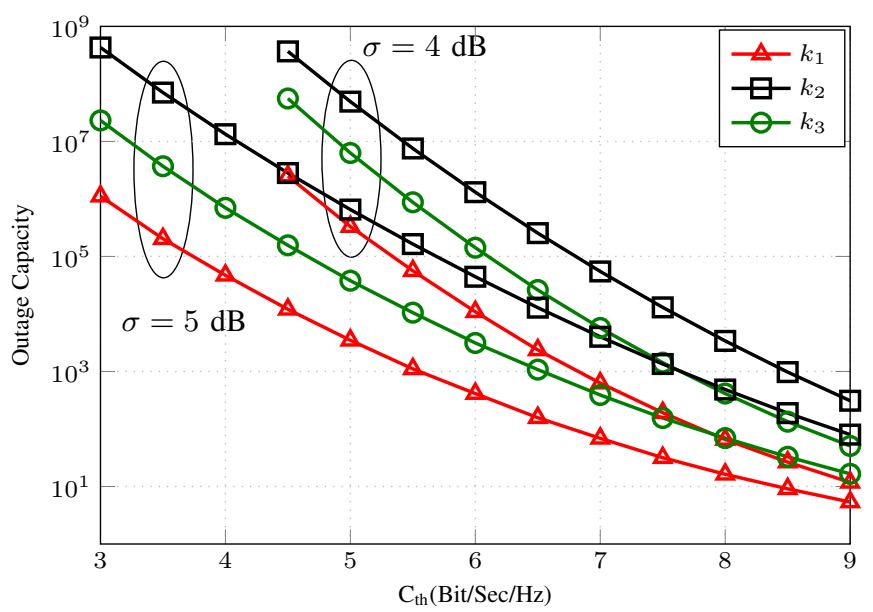

Fig. 4. (a) Outage capacity of $N=4$-branch MRC diversity receivers over i.i.d Log-normal fading variates with $E_{s} / N_{0}=10 \mathrm{~dB}$, and $\mu=10 \mathrm{~dB}$. (b) Efficiency $k_{i}, i=1,2,3$, with same parameters. Number of simulation runs: $M=10^{6}$.

\section{B. Nakagami Fading with EGC Diversity Technique}

In a second experiment, we consider the evaluation of the $\mathrm{OC}$ at the output of the EGC receiver when operating over the Nakagami fading for different number of combiners. We recall that the PDF of the Nakagami envelope $R_{i}, i=1,2, \ldots, N$, is given by

$$
f_{R_{i}}(r)=\frac{2 m_{i}^{m_{i}}}{\Gamma\left(m_{i}\right) \Omega_{i}^{m_{i}}} r^{2 m_{i}-1} \exp \left(-\frac{m_{i}}{\Omega_{i}} r^{2}\right), r>0,
$$

where $m_{i}$ and $\Omega_{i} i=1,2, \ldots, N$, denote respectively the shape and the spread parameters, whereas $\Gamma(\cdot)$ is the Gamma function. Interestingly, provided that $m>1 / 2$, the CDF of a Nakagami distribution is log-concave [31]. Hence under 
the i.i.d setting, $Y_{i}^{*}=-\gamma_{t h} / N$ is the solution of $\left(P^{\prime}\right)$. Consequently, a closed-form expression for $\theta_{1}^{*}$ in (28) is obtained by:

$$
\theta_{1}^{*}=1-\frac{1}{\Lambda_{Y_{1}}\left(-\gamma_{t h} / N\right)},
$$

$\Lambda_{Y_{1}}(\cdot)$ being the hazard function of $Y_{1}, Y_{2}, \ldots, Y_{N}$ given by:

$$
\Lambda_{Y_{1}}(y)=-\log \left(\frac{\gamma\left(m, m y^{2} / \Omega\right)}{\Gamma(m)}\right),
$$

where $\Gamma(\cdot, \cdot)$ and $\gamma(\cdot)$ denote respectively the lower incomplete gamma function and the Gamma function [32]. As for the twisting parameter $\theta_{2}^{*}$ used in the second IS approach, its value is given in (62) and is equal for this particular case to:

$$
\theta_{2}^{*}=1+\frac{N}{\log \left(1-\frac{\gamma\left(m, m \gamma_{t h}^{2} / \Omega\right)}{\Gamma(m)}\right)}
$$

In Table II, we investigate the ability of the two proposed IS schemes in generating important samples, i.e, samples that belong to the desired set $\left\{C_{\gamma_{e n d}}<C_{t h}\right\}$. To this end, we compute the percentage of such samples when using naive MC simulations and the two proposed IS schemes (we refer them to IS1 and IS2 in the table) as a function of the probability of interest and for a number of simulation runs $M=10^{6}$. We easily observe from this table that, contrary to the naive MC method which fails to generate important values as we decrease the probability of interest, the two proposed IS approaches continue to generate samples in the desired region even for very small probabilities.

TABLE II

PERCENTAGE OF IMPORTANT SAMPLES FOR $N=4$-BRANCH EGC DIVERSITY RECEIVERS OVER I.I.D NAKAGAMI FADING VARIATES WITH $E_{s} / N_{0}=10 \mathrm{DB}, m=0.8, \Omega=8$, AND $M=10^{6}$.

\begin{tabular}{|l|l|c|c|c|c|}
\hline$C_{t h}$ & $\begin{array}{l}\text { Naive } \\
\text { MC }\end{array}$ & $\hat{\alpha}_{I S, 1}$ & $I S 1$ & $\hat{\alpha}_{I S, 2}$ & $I S 2$ \\
\hline 4 & $0.058 \%$ & $5.97 \times 10^{-4}$ & $10.415 \%$ & $5.96 \times 10^{-4}$ & $11.254 \%$ \\
3 & $0.005 \%$ & $5.68 \times 10^{-5}$ & $8.789 \%$ & $5.69 \times 10^{-5}$ & $12.216 \%$ \\
2 & $0.0006 \%$ & $3.86 \times 10^{-6}$ & $7.438 \%$ & $3.96 \times 10^{-6}$ & $13.051 \%$ \\
1 & $0 \%$ & $1.19 \times 10^{-7}$ & $6.263 \%$ & $1.20 \times 10^{-7}$ & $13.608 \%$ \\
0.5 & $0 \%$ & $7.32 \times 10^{-9}$ & $5.610 \%$ & $7.20 \times 10^{-9}$ & $13.933 \%$ \\
\hline
\end{tabular}

Next, we plot in Fig. 5.a the OC evaluated by the naive $\mathrm{MC}$ and the two proposed IS schemes. We notice again the failure of naive MC simulations in estimating small OC. In fig. 5.b, we plot the amounts of variance reductions achieved by the two proposed IS schemes. We observe that the use of the second IS scheme for instance can indeed save up to $10^{7}$ times the number of simulation runs needed by naive MC simulations while keeping the same accuracy requirement. Furthermore, it is worth pointing out that it is the second IS estimator that achieves the highest performance, with a gain that becomes higher as the threshold decreases. For instance, at a threshold value of $C_{t h}=0.5 \mathrm{Bit} / \mathrm{Sec} / \mathrm{Hz}$ and when $N=4$, the second IS estimator requires $10^{2}$ times less simulation runs than the first one. Such a result is expected and likely owes to the bounded relative error property that enjoys the second IS estimator, the first IS estimator satisfying only a weaker property, that is the asymptotic optimality criterion. (a)

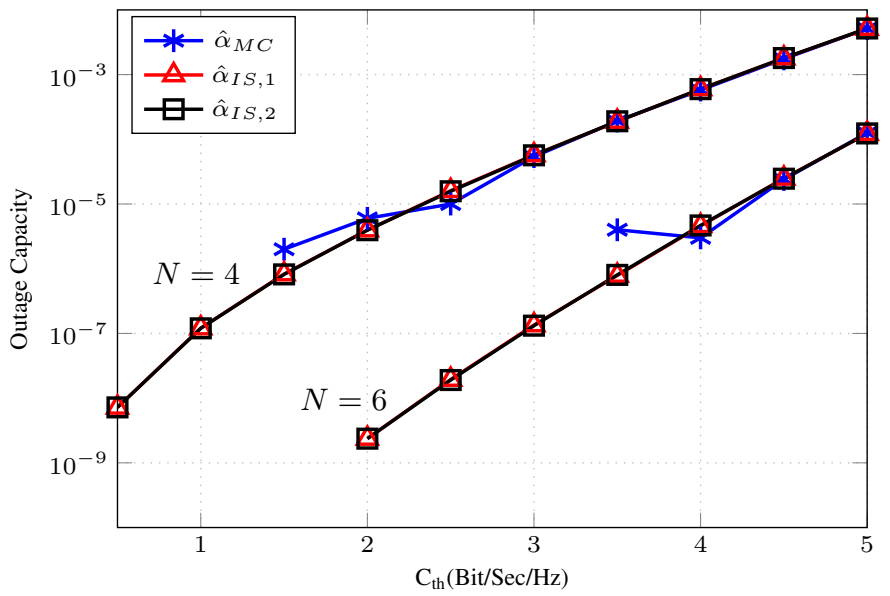

(b)

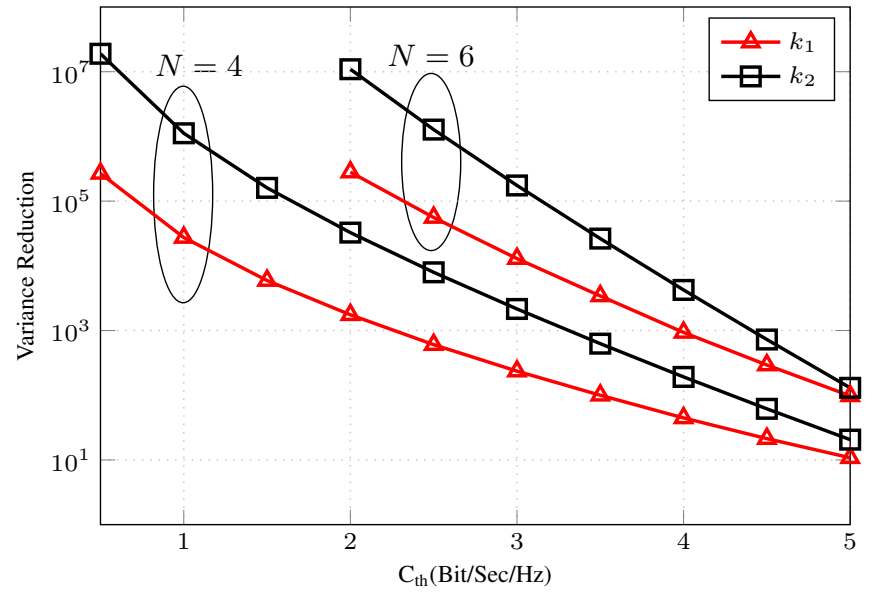

Fig. 5. (a) Outage capacity of $N$-branch EGC diversity receivers over i.i.d Nakagami fading variates with $E_{s} / N_{0}=10 \mathrm{~dB}, \Omega=8$, and $m=0.8$. (b) Efficiency $k_{i}, i=1,2$, with same parameters. Number of simulation runs: $M=10^{6}$.

\section{CONClusion}

We consider in this paper the estimation of the CDF for the sum of independent RVs. Such a question is driven by the problem of evaluating the OC of MRC/EGC diversity techniques over generalized fading channels. It is now stirring an increasing interest in the field of rare event simulation algorithms, the focus of which is shifting away from the estimation of the right tail of the sum distribution to consideration of the left tail region.

Building upon the framework of rare event simulation techniques, we propose two unified, yet efficient, hazard rate twisting IS-based simulation approaches that efficiently estimate the $\mathrm{CDF}$ of the sum of independent RVs. The two proposed schemes achieve higher gain over the naive MC simulations but present different characteristics. More specifically, the first IS approach applies to arbitrary distributions and was shown to achieve the asymptotic optimality criterion for arbitrary independent fading models. On the other hand, the second one applies only to the i.i.d. case but achieves the asymptotic optimality criterion for the Log-normal setting and the welldesired bounded relative criterion for the majority of the 
well-known distributions. Simulations results supporting our theoretical findings were provided. They have in particular illustrated the substantial computational efficiency achieved by these IS schemes over naive MC simulations.

\section{REFERENCES}

[1] N. Ben Rached, A. Kammoun, M.-S. Alouini, and R. Tempone, "A unified simulation approach for the fast outage capacity evaluation over generalized fading channels," in Proc. of the IEEE International Symposium on Information Theory (ISIT'2015), Jun. 2015, pp. 346-350.

[2] M.K. Simon and M.-S. Alouini, Digital Communication over Fading Channels, 2nd Edition. New York: Wiley, 2004.

[3] G. L. Stüber, Principles of Mobile Communication, 2nd Edition. Norwell, MA, USA: Kluwer Academic Publishers, 2001.

[4] S. M. Navidpour, M. Uysal, and M. Kavehrad, "BER performance of free-space optical transmission with spatial diversity." IEEE Transactions on Wireless Communications, vol. 6, no. 8, pp. 2813-2819, Aug. 2007.

[5] H. Sandalidis, T. Tsiftsis, G. Karagiannidis, and M. Uysal, "Ber performance of FSO links over strong atmospheric turbulence channels with pointing errors," IEEE Communications Letters, vol. 12, no. 1, pp. 4446, Jan. 2008.

[6] F. Babich and G. Lombardi, "Statistical analysis and characterization of the indoor propagation channel," IEEE Transactions on Communications, vol. 48, no. 3, pp. 455-464, Mar. 2000.

[7] N. Sagias and G. Karagiannidis, "Gaussian class multivariate Weibull distributions: Theory and applications in fading channels," IEEE Transactions on Information Theory, vol. 51, no. 10, pp. 3608-3619, Oct. 2005.

[8] N. Beaulieu and Q. Xie, "An optimal Lognormal approximation to Lognormal sum distributions," IEEE Transactions on Vehicular Technology, vol. 53, no. 2, pp. 479-489, Mar. 2004.

[9] M. Di Renzo, F. Graziosi, and F. Santucci, "Further results on the approximation of Log-normal power sum via Pearson type IV distribution: a general formula for log-moments computation," IEEE Transactions on Communications, vol. 57, no. 4, pp. 893-898, Apr. 2009.

[10] J. Filho and M. Yacoub, "Simple precise approximations to Weibull sums," IEEE Communications Letters, vol. 10, no. 8, pp. 614-616, Aug. 2006.

[11] D. da Costa, M. Yacoub, and J. Filho, "An improved closed-form approximation to the sum of arbitrary Nakagami- m variates," IEEE Transactions on Vehicular Technology, vol. 57, no. 6, pp. 3854-3858, Nov. 2008.

[12] K. Peppas, "A simple, accurate approximation to the sum of GammaGamma variates and applications in MIMO free-space optical systems," IEEE Photonics Technology Letters, vol. 23, no. 13, pp. 839-841, Jul. 2011.

[13] J. Hu and N. Beaulieu, "Accurate simple closed-form approximations to Rayleigh sum distributions and densities," IEEE Communications Letters, vol. 9, no. 2, pp. 109-111, Feb. 2005.

[14] N. Beaulieu and F. Rajwani, "Highly accurate simple closed-form approximations to Lognormal sum distributions and densities," IEEE Communications Letters, vol. 8, no. 12, pp. 709-711, Dec. 2004.

[15] M. Di Renzo, L. Imbriglio, F. Graziosi, and F. Santucci, "Smolyak's algorithm: A simple and accurate framework for the analysis of correlated Log-normal power-sums," IEEE Communications Letters, vol. 13, no. 9 , pp. $673-675$, Sep. 2009.

[16] D. Senaratne and C. Tellambura, "Numerical computation of the Lognormal sum distribution," in Proc. of the IEEE Global Telecommunications Conference (GLOBECOM'2009), Honolulu, Hawaii, US, Nov. 2009, pp. $1-6$.

[17] - , "A general numerical method for computing the probability of outage," in Proc. of the IEEE Wireless Communications and Networking Conference (WCNC'09), Apr. 2009, pp. 1-6.

[18] S. Asmussen, J. L. Jensen, and L. Rojas-Nandayapa, "Exponential family techniques for the Lognormal left tail," arXiv preprint arXiv:1403.4689, 2014.

[19] A. Gulisashvili and P. Tankov, "Tail behavior of sums and differences of Log-normal random variables," arXiv preprint arXiv:1309.3057, 2013.

[20] J. Sadowsky, "On the optimality and stability of exponential twisting in Monte Carlo estimation," IEEE Transactions on Information Theory, vol. 39, no. 1, pp. 119-128, Jan. 1993.

[21] J. Sadowsky and J. Bucklew, "On large deviations theory and asymptotically efficient Monte Carlo estimation," IEEE Transactions on Information Theory, vol. 36, no. 3, pp. 579-588, May. 1990.
[22] S. Asmussen, J. Jensen, and L. Rojas-Nandayapa, "On the Laplace transform of the Lognormal distribution," Methodology and Computing in Applied Probability, pp. 1-18, 2014.

[23] S. Juneja and P. Shahabuddin, "Simulating heavy tailed processes using delayed hazard rate twisting," ACM Trans. Model. Comput. Simul., vol. 12, no. 2, pp. 94-118, Apr. 2002.

[24] N. Ben Rached, F. Benkhelifa, M.-S. Alouini, and R. Tempone, "A fast simulation method for the Log-normal sum distribution using a hazard rate twisting technique," in Proc. of the IEEE International Conference on Communications (ICC'2015), Jun. 2015, pp. 4259-4264.

[25] N. Ben Rached, F. Benkhelifa, A. Kammoun, M.-S. Alouini, and R. Tempone, "Additional results on the hazard rate twisting-based simulation approach," 2014. [Online]. Available: http://sri-uq.kaust.edu.sa/Pages/ Page_BenRached_Benkhelifa_Kammoun_Alouini_Tempone_1.aspx

[26] N. Ben Rached, A. Kammoun, M.-S. Alouini, and R. Tempone, "An improved hazard rate twisting approach for the statistic of the sum of subexponential variates," IEEE Communications Letters, vol. 19, no. 1, pp. 14-17, Jan 2015.

[27] J. A. Bucklew, Introduction to Rare Event Simulation, ser. Springer series in statistics. New York: Springer, 2004.

[28] F. Yilmaz and M.-S. Alouini, "A unified MGF-based capacity analysis of diversity combiners over generalized fading channels," IEEE Transactions on Communications, vol. 60, no. 3, pp. 862-875, Mar. 2012.

[29] P.M. Shankar, Fading and Shadowing in Wireless Systems. Springer New York, 2012.

[30] S. Asmussen and P. W. Glynn, Stochastic simulation : algorithms and analysis, ser. Stochastic modelling and applied probability. New York: Springer, 2007.

[31] M. Bagnoli and T. Bergstrom, "Log-concave probability and its applications," Economic Theory, vol. 26, no. 2, pp. 445-469, 2005.

[32] I. S. Gradshteyn and I. M. Ryzhik, Table of integrals, series, and products, 7th ed. Elsevier/Academic Press, Amsterdam, 2007.

[33] S. András, A. Baricz, and Y. Sun, "The generalized Marcum Q- function: an orthogonal polynomial approach," Acta Universitatis Sapientiae Mathematica, vol. 3, no. 1, pp. 60-76, 2011

[34] A. Kilbas and M. Saigo, H-Transforms : Theory and Applications (Analytical Method and Special Function), 1st ed. CRC Press, 2004.

[35] S. Asmussen, J. H. Blanchet, S. Juneja, and L. Rojas-Nandayapa, "Efficient simulation of tail probabilities of sums of correlated Lognormals," Annals OR, vol. 189, no. 1, pp. 5-23, 2011.

[36] L. Devroye, Non-Uniform Random Variate Generation. New York: Springer-Verlag, 1986.

[37] D. P. Kroese, T. Taimre, and Z. I. Botev, Handbook of Monte Carlo methods. Hoboken, N.J. Wiley, 2011.

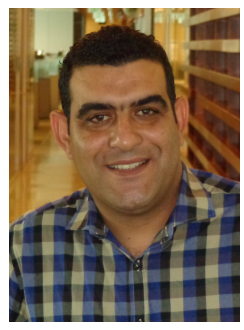

Nadhir Ben Rached was born in Nabeul, Tunisia He received the Diplôme d'Ingénieur degree from the École Polytechnique de Tunisie, La Marsa, Tunisia, in 2012 and the M.S. degree in Applied Mathematics and Computational Science from King Abdullah University of Science and Technology, Thuwal, Saudi Arabia, in 2013, where he is currently working toward the Ph.D degree in Statistics. His current research interests include rare event simulation algorithms for the accurate performance analysis of wireless communication systems.

Abla Kammoun was born in Sfax, Tunisia. She received the engineering degree in signal and systems from the Tunisia Polytechnic School, La Marsa, and the Master's degree and the Ph.D. degree in digital communications from Télécom Paris Tech [then École Nationale Supérieure des Télécommunications (ENST)]. From June 2010 to April 2012, she has been a Postdoctoral Researcher in the TSI Department, Télécom Paris Tech. Then she has been at Supèlec at the Alcatel-Lucent Chair on Flexible Radio until December 2013. Currently, she is a research scientist at KAUST university. Her research interests include performance analysis, random matrix theory, and semi-blind channel estimation. She is the recipient of the SAM 2014 second Prize Best paper award. 


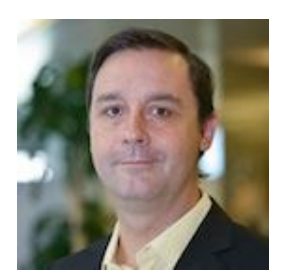

Raul Tempone was born in Montevideo, Uruguay, in 1969. He received the B.E. degree in industrial engineer at the University of the Republic, Montevideo, Uruguay in 1995. After his graduation he worked on the optimal dispatch of electricity for the Uruguayan system using techniques from nonlinear stochastic programming and visited the Royal Institute of Technology (KTH) in Stockholm, Sweden, to study further numerical analysis. He obtained a MSc in Engineering Mathematics in 1999 (inverse problems for incompressible flows, supervised by Jesper Oppelstrup, KTH) and a $\mathrm{PhD}$ in Numerical Analysis in 2002 (a posteriori error estimation and control for stochastic differential equations, supervised by Anders Szepessy, KTH). He later moved to ICES, UT Austin, to work as a postdoc from 2003 until 2005 in the area of numerical methods for PDEs with random coefficients (supervised by Ivo Babuska). In 2005 he became an assistant professor with the School of Computational Sciences and the Department of Mathematics at Florida State University, Tallahassee. In 2007 he was awarded the first Dahlquist fellowship by KTH and COMSOL for his contributions to the field of numerical approximation of deterministic and stochastic differential equations. In 2009 he joined KAUST as an Associate Professor in Applied Mathematics (founding faculty). He later became there the Director of the KAUST Center for Uncertainty Quantification and was promoted to Full Professor in 2015.

Dr. Tempone's research interests are in the mathematical foundation of computational science and engineering. More specifically, he has focused on a posteriori error approximation and related adaptive algorithms for numerical solutions of various differential equations, including ordinary differential equations, partial differential equations, and stochastic differential equations. $\mathrm{He}$ is also interested in the development and analysis of efficient numerical methods for uncertainty quantification and Bayesian model validation. The areas of application he considers include, among others, engineering, chemistry, biology, physics as well as social science and computational finance.

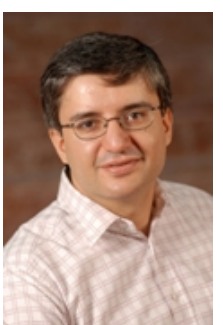

Mohamed-Slim Alouini (S'94, M'98, SM'03, F'09) was born in Tunis, Tunisia. He received the Ph.D. degree in Electrical Engineering from the California Institute of Technology (Caltech), Pasadena, CA, USA, in 1998. He served as a faculty member in the University of Minnesota, Minneapolis, MN, USA, then in the Texas A\&M University at Qatar, Education City, Doha, Qatar before joining King Abdullah University of Science and Technology (KAUST), Thuwal, Makkah Province, Saudi Arabia as a Professor of Electrical Engineering in 2009. His current research interests include the modeling, design, and performance analysis of wireless communication systems. 